\title{
Amino Acid Transporter (AAT) Gene Family In Foxtail Millet (Setaria Italica L.): Widespread Family Expansion, Functional Differentiation, Roles In Quality Formation And Response To Abiotic Stresses
}

\author{
Yang Yang \\ Northwest A\&F University: Northwest Agriculture and Forestry University \\ Yongmao Chai \\ Northwest A\&F University: Northwest Agriculture and Forestry University \\ Jiayi Liu \\ Northwest A\&F University: Northwest Agriculture and Forestry University \\ Jie Zheng \\ Northwest A\&F University: Northwest Agriculture and Forestry University \\ Zhangchen Zhao \\ Northwest A\&F University: Northwest Agriculture and Forestry University \\ Amo Aduragbemi \\ Northwest A\&F University: Northwest Agriculture and Forestry University \\ Chunge Cui \\ Northwest A\&F University: Northwest Agriculture and Forestry University \\ Qiumei Lu \\ Northwest A\&F University: Northwest Agriculture and Forestry University \\ Liang Chen \\ Northwest A\&F University: Northwest Agriculture and Forestry University \\ Yin-Gang Hu ( $\sim$ huyingang@nwsuaf.edu.cn ) \\ Northwest Agriculture and Forestry University https://orcid.org/0000-0002-1831-6375
}

Research article

Keywords: Foxtail millet, amino acid transporter, genome-wide characterization, functional differentiation, grain quality, abiotic stresses

Posted Date: December 4th, 2020

DOl: https://doi.org/10.21203/rs.3.rs-119005/v1

License: () (1) This work is licensed under a Creative Commons Attribution 4.0 International License. Read Full License

Version of Record: A version of this preprint was published at BMC Genomics on July 8th, 2021. See the published version at https://doi.org/10.1186/s12864021-07779-9. 


\section{Abstract}

Background: Amino acid transporters (AATs) plays an essential roles in growth and development of plants, including amino acids long-range transport, seed germination, quality formation, responsiveness to pathogenic bacteria and abiotic stress by modulating the transmembrane transfer of amino acids. In this study, we performed a genome-wide screening to analyze the $A A T$ genes in foxtail millet (Setaria italica L.), especially those associated with quality formation and abiotic stresses response.

Results: A total number of $94 A A T$ genes were identified and divided into 12 subfamilies by their sequence characteristics and phylogenetic relationship. A large number $(58 / 94,62 \%)$ of $A A T$ genes in foxtail millet were expanded via gene duplication, involving 13 tandem and 12 segmental duplication events. Tandemly duplicated genes had a significant impact on their functional differentiation via sequence variation, structural variation and expression variation. Further comparison in multiple species showed that in addition to paralogous genes, the expression variations of the orthologous $A A T$ genes also contributed to their functional differentiation. The transcriptomic comparison of two millet cultivars verified the direct contribution of the AAT genes such as SiAAP1, SiAAP8, and SIAUX2 in the formation of grain quality. In addition, the qRT-PCR analysis suggested that several $A A T$ genes continuously responded to diverse abiotic stresses, such as SIATLb1, SiANT1. Finally, combined with the previous studies and analysis on sequence characteristics and expression patterns of $A A T$ genes, the possible functions of the foxtail millet $A A T$ genes were predicted.

Conclusion: This study for the first time reported the evolutionary features, functional differentiation, roles in the quality formation and response to abiotic stresses of foxtail millet $A A T$ gene family, thus providing a framework for further functional analysis of SiAAT genes, and also contributing to the applications of $A A T$ genes in improving the quality and resistance to abiotic stresses of foxtail millet, and other cereal crops.

\section{Background}

Amino acids, as small-molecule organic compounds containing nitrogen, are the main carriers of nitrogen exchange in plants and the nutrients necessary for plant growth, development and quality [1]. The absorption and transport of amino acids are mainly mediated by amino acid transporters (AATs) [2]. Through the transmembrane transport of amino acids, amino acid transporters play essential roles in plant growth and development, including seed germination [3, 4], long-distance transport of amino acids [5], quality formation [6, 7], and response to pathogenic bacteria [8] and abiotic stress [9]. A total of 72, 65, 189, 85 and 297 amino acid transporters have been characterized in potato [10], Arabidopsis [1], soybean [11], rice [12] and wheat [13], respectively, suggesting that amino acid transporters are widely present in higher plants.

The AAT gene family in plants belongs to APC transporter superfamily, that comprises of two main families of amino acid/auxin permease (AAAP) family and amino acid-polyamine-choline (APC) transporter family. The AAAP family consists of Amino acid permeases (AAPs), lysine and histidine transporters (LHTs), auxin transporters (AUXs), c-aminobutyric acid transporters (GATs), neutral amino acid transporters (ANTs), proline transporters (ProTs), and amino acid transporter-like a/b (ATLa/b) proteins $[8,14,15]$. The APC family comprises cationic amino acid transporters (CATs), amino acid/choline transporters (ACTs), polyamine $\mathrm{H}^{+}$-symporters (PHSs), and tyrosine-specific transporters (TTPs) subfamilies [10, 16].

Based on the heterologous expression system, the functions of $A A T$ genes in Arabidopsis have been characterized in detail [17]. There are 8 AAP genes (AtAAP1-AtAAP8), and 6 AtAAP genes transport charged and neutral amino acids with various affinities [17, 18]. In endosperm, roots and cotyledon, AtAAP1 is abundantly expressed, which is essential for the synthesis of storage proteins in Arabidopsis seed and seed yield, while regulating embryonic development and amino acid input in roots $[14,19]$. AtAAP2 and AtAAP3 are mainly involved in the xylem-phloem transfer of amino acids and the absorption of amino acids in xylem, respectively $[20,21]$. AtAAP4 and AtAAP5 can transfer amino acids to developing embryos and AtAAP5 plays crucial role in the root amino acid intake system [22]. AtAAP6 affects the interaction between plants and aphids through the regulation of the phloem's amino acid composition [8]. AtAAP8 is mainly responsible for transporting amino acids into the endosperm and embryo at the early embryonic development [23]. Additionally, the functions of some $A A P s$ in other species have also been explored. In Vicia faba, VfAAP1 is expressed abundantly in cotyledons and other pool tissues, and significantly affects the storage protein content (GPC) in seeds by regulating the nitrogen content of seeds [4, 6], while VfAAP3 is significantly enriched in roots, stems, pods, pistils and seed coats at various stages of development [4]. In potato, StAAP1 is expressed during the source-sink transition process, and the free amino acid contents are decreased in transgenic plants with reduced StAAP1 expression [24], and the expression patterns of StAAP4, 5, and 6 may be associated with the long-distance transport of amino acids [10]. In barley, HVAAP2 and 6 are mainly expressed in nutritive organs, while HvAAP3 is high-expressed in seeds and contributes significantly to nitrogen accommodate in developing seed [25]. In rice, OSAAP1 affects plant growth and grain yield by modulating the redistribution of neutral amino acids [26], and the quantitative trait locus qPC1 (QTL) modulation of the rice GPC is linked to the expression of OsAAP6 [7]. In addition, several wheat TaAAP genes have been confirmed to co-localize with QTL of grain protein content [27].

In addition to the in-depth studies on AAP genes, the functions of some genes in other subfamilies have also been explored [5, 8, 9]. AtLHT1 plays an indispensable role in the uptake of amino acids and mesophyll formation in root, while AtLHT2 mainly involves in the movements and allocation of amino acids in floral organs [28-30]. AtANT1 is expressed abundantly in flowers and stem leaves, transporting neutral and aromatic amino acids as well as indole-3acetic acid (IAA) and arginine [31]. AtAUX1 and AtLAX3 affect the root gravitropism and lateral root formation by facilitating IAA sink-source distribution and auxin-induced expression of root cell-wall-remodeling genes, respectively [32-35]. AtProT1 and AtProT2 participate in the long-distance transportation of proline and the absorption of proline and glycine betaine in roots of Arabidopsis, respectively [36, 37]. AtProT3 is mainly expressed in leaf epidermal cells, which may be involved in the regulation of proline distribution in leaves [38]. AtCAT5 mediates the reabsorption of amino acids permeated at leaf margin by transporting basic amino acids, while AtCAT3, AtCAT6 and AtCAT8 prefer to transport acidic or neutral amino acids [17]. AtBAT1 has been identified as a bidirectional amino acid transporter expressed on mitochondrial membrane, which mediates $\gamma$-aminobutyric acid (GABA) from cytosol to mitochondria [39].

In addition to the role of $A A T$ genes in seed germination, long-distance amino acid transport, root gravitropism, the formation of lateral roots and reproductive organs, a number of AAT genes play an important role in reducing damage to plants under various abiotic stresses, specifically by promoting the

Page $2 / 37$ 
transportation of stress-response compounds and compatible solutes such as proline, GABA, and betaine [40]. For example, the ProT genes rapidly distribute proline to minimize damage to plants caused by water shortage [41]. AtProT2 is strongly induced by salt stress, and plays a role in nitrogen distribution under salt stress [42]. GmProT1 and GmProT2 are sensitive to stress in the roots and transgenic plants can transport more proline after spraying proline [43]. AtGAT1 is significantly induced by GABA enrichment in plants under mechanical damage, stress or sensitive environment [44]. OSAAP6, OsAAP11 and OsANT3 are strongly up-regulated under salt and drought stresses, to regulate the tolerance to stress of plants through the transport of stress compounds [12].

Foxtail millet (Setaria italica L.), a member of the Poaceae family, is one of the oldest cereal crops, domesticated in Northern China. It is rich in essential amino acids, fatty acids and minerals, which is of important significance to human health [45]. To date, the $A A T$ gene family in foxtail millet has not been characterized. Given their essential roles in the growth and grain quality formation of foxtail millet, we carried out a detailed characterization of $A A T$ genes in foxtail millet. The aims of this study are : (a) Accurately identify and characterize $A A T$ genes in foxtail millet based on the latest genome sequence; (b) Explore the roles of paralogous and orthologous $A A T$ genes in the build-up and functional differentiation of AAT family in foxtail millet through comparative analysis of their gene structure, sequence feature and expression patterns; (c) Mine the key candidate $A A T$ genes that affect grain quality formation of foxtail millet through the comparative transcriptome analysis of two genotypes; (d) Detect the response of SiAAT genes to diverse abiotic stresses by Quantitative RealTime PCR; (e) Predict the possible functions of the SiAAT genes based on the previous studies, sequence composition and expression pattern. This work will provide a deeper insight and understanding of the $A A T$ genes in foxtail millet. Also, important clues for their functional analysis and applications in improving quality and resistances to abiotic stresses will be provided.

\section{Results}

\section{The foxtail millet genome contains 94 AAT genes, which all with transmembrane regions}

Firstly, 104 putative $A A T$ transcripts were identified in foxtail millet based on local BLASP. However, 94 high-confidence non-redundant $A A T$ genes were confirmed after screening the conservative domains by HMMER, CDD and Interpro databases and removing the different transcripts of the same gene and sequences containing incomplete conserved domains, which was basically at the same level as other gramineous species besides hexaploid wheat (Table 1 ; Additional file 5: Table S1). Except for tetraploid soybean, AAT gene family in foxtail millet was expanded than that in Arabidopsis and potato. Overall, the number of $A A T$ genes in monocots was higher than that in dicots. The $A A T$ genes identified in foxtail millet were renamed according to their chromosomal localizations and phylogenetic relationships with other species. The length of AAT proteins in foxtail millet ranged from 311 to $984 a a$, with molecular weight $(\mathrm{Mw})$ varing from 4.97 to $107.5 \mathrm{kD}$, and isoelectric point (pl) ranged from 4.94 to 9.99 (Additional file 5: Table S1). Different subfamilies of $A A T$ genes showed abundant diversity in subcellular localization, with the subfamily members of AAP, LHT, GAT, AUX and ProT all located on plasma membrane, while those of TTP, ACT, ANT and ATLb all located on vacuole membrane. Some members of the same subfamily showed different subcellular localization, such as, different genes of the ATLa subfamily located on both membranes of plasma and vacuole, while that of the CAT and PHS subfamilies located on the three membranes of the plasma, vacuole and chloroplast.

Table 1

Comparison on the gene abundance of twelve subfamilies of $A A T$ genes in 6 monocots and 3 eudicots.

\begin{tabular}{|c|c|c|c|c|c|c|c|c|c|c|}
\hline & & \multicolumn{6}{|l|}{ Monocots } & \multicolumn{3}{|l|}{ Eudicots } \\
\hline & & Millet $^{a}$ & Sorghum & Maize & Brachypodium & Rice & Wheat & Soybean & Arabidopsis & Potato \\
\hline \multirow[t]{8}{*}{ AAAP } & AAP & $20(21.27 \%)$ & $17(22.07 \%)$ & $24(22.43 \%)$ & 19(23.75\%) & $19(22.35 \%)$ & $66(22.30 \%)$ & $35(18.52 \%)$ & $8(12.70 \%)$ & $8(11.11 \%)$ \\
\hline & LHT & $12(12.77 \%)$ & $8(10.39 \%)$ & $15(14.42 \%)$ & $8(10.00 \%)$ & $6(7.05 \%)$ & $24(8.11 \%)$ & $24(12.70 \%)$ & $10(15.87 \%)$ & $11(15.28 \%)$ \\
\hline & GAT & $6(6.38 \%)$ & $4(5.19 \%)$ & $2(1.92 \%)$ & $3(3.75 \%)$ & $4(4.71 \%)$ & $14(4.73 \%)$ & 19(10.05\%) & $2(3.17 \%)$ & $3(4.17 \%)$ \\
\hline & ProT & $1(1.06 \%)$ & $1(1.30 \%)$ & $2(1.92 \%)$ & $2(2.50 \%)$ & $3(3.53 \%)$ & $9(3.04 \%)$ & $7(3.70 \%)$ & $3(4.76 \%)$ & $4(6.35 \%)$ \\
\hline & AUX & $4(4.25 \%)$ & $5(6.49 \%)$ & $6(5.60 \%)$ & $3(3.75 \%)$ & $5(5.88 \%)$ & $15(5.07 \%)$ & $16(8.47 \%)$ & $4(6.35 \%)$ & $5(7.94 \%)$ \\
\hline & ATLa & $6(6.38 \%)$ & $6(7.79 \%)$ & $7(6.54 \%)$ & $6(7.50 \%)$ & $7(8.24 \%)$ & $18(6.08 \%)$ & $16(8.47 \%)$ & $5(7.94 \%)$ & $8(11.11 \%)$ \\
\hline & ANT & $2(2.13 \%)$ & $4(5.19 \%)$ & $3(2.8 \%)$ & $5(6.25 \%)$ & $4(4.71 \%)$ & $18(6.08 \%)$ & $6(3.17 \%)$ & $4(6.34 \%)$ & $5(7.94 \%)$ \\
\hline & ATLb & $14(14.89 \%)$ & $8(10.39 \%)$ & 17(15.89\%) & $7(8.75 \%)$ & $10(11.76 \%)$ & $40(13.51 \%)$ & $30(15.87 \%)$ & 10(15.87\%) & $8(11.11 \%)$ \\
\hline \multirow[t]{4}{*}{ APC } & ACT & $8(8.51 \%)$ & $5(6.49 \%)$ & $7(6.54 \%)$ & $6(7.50 \%)$ & $7(8.24 \%)$ & $21(7.09 \%)$ & $7(3.70 \%)$ & $1(1.59 \%)$ & $1(1.39 \%)$ \\
\hline & CAT & $12(12.77 \%)$ & $11(14.29 \%)$ & 14(13.08\%) & $11(13.75 \%)$ & $11(12.94 \%)$ & $31(10.47 \%)$ & $19(10.05 \%)$ & $9(14.29 \%)$ & $9(12.5 \%)$ \\
\hline & PHS & $8(8.51 \%)$ & $7(9.09 \%)$ & $7(6.54 \%)$ & $7(8.75 \%)$ & $9(10.59 \%)$ & $31(10.47 \%)$ & $9(4.76 \%)$ & $5(7.94 \%)$ & $8(11.11 \%)$ \\
\hline & TTP & $1(1.06 \%)$ & $1(1.30 \%)$ & $3(2.80 \%)$ & $3(3.75 \%)$ & $0(0.00 \%)$ & $9(3.04 \%)$ & $1(0.53 \%)$ & 2(3.17\%) & $2(2.78 \%)$ \\
\hline Total & & 94 & 77 & 107 & 80 & 85 & 296 & 189 & 63 & 72 \\
\hline
\end{tabular}

a The numbers represent the number of identified AAT subfamily members, and the percentages in the brackets represent the proportion to all $A A T$ genes.

The number of predicted TM regions in SiAATs varied in different subfamilies, which ranged from 7 to 14 (Fig. 1a; Additional file 5: Table S1). For example, all AUXs contained 10 TM regions, ATLas contained 10 or 11 TM regions, while CAT and PHS subfamilies ranged from 9 to 14 and 7 to 12 , respectively. 
Here, 93 of the 94 SiAATs were mapped to 9 chromosomes. The remaining one was mapped to an unassembled scafford (Additional file 1: Figure S1), which were unevenly distributed, with 18 on chromosome 7 and 2 on chromosome 2 as the most and least, respectively. The apparent regional enrichment of SiAATs on some chromosomes was observed on their distributions. For instance, a large number of SiAATs were located on the ends of chromosomes 1,5 , and 7. Moreover, some SiAATs were mainly concentrated at the fronts of chromosomes 6 and 8 (Additional file 1: Figure S1).

Of the $94 A A T$ genes in foxtail millet, 58 (62\%) were involved in gene duplication events, including 36 tandem and 25 segmental duplications, of which SiATLb12, SiLHT2 and SiLHT9 were involved in both duplications (Additional file 1: Figure S1; Additional file 5: Table S1). The 36 tandem duplications were classified into 13 groups, of which 2 groups with 5 genes (TD10, TD11), 1 group with 4 genes (TD3), 2 groups with 3 genes (TD1, TD13), and the remaining 8 groups all with 2 genes (Additional file 5: Table S1). The 25 segmental duplications were classified into 12 groups, except for SD3 containing 3 genes, with other possessing 2 genes. Gene duplication events produced a large number of paralogous AAT genes, thus promoting the significant build-up of the $A A T$ gene family in foxtail millet.

\section{The phylogenetic tree and structure of AAT genes in foxtail millet}

The phylogenetic analysis with the AAT protein sequences from foxtail millet (94), rice (85), Arabidopsis (63) and potato (72) showed that they could be divided into twelve genetic groups with high confidence and that from Monocots and Eudicots distributed in the same group (Fig. 2). The 65 SiAAT proteins of the AAAP family were divided into 8 subfamilies of amino acid permeases (AAPs, 20), lysine, histidine transporters (LHTs, 12), GABA transporters (GATs), proline transporters (ProTs, 1), aux transporters (AUXs, 4), amino acid transporter-like a (ATLa, 6), aromatic and neutral amino acid transporters (ANTs, 2) and amino acid transporter-like $b(A T L b, 14)$. The 29 SiAAT proteins of the APC family were clearly distinguished into 4 subfamilies of cationic amino acid transporters (CATs, 12), amino acid/choline transporters (ACTs, 8), tyrosine-specific transporters (TTPs, 1), and polyamine $\mathrm{H}^{\natural}$-symporters (PHSs, 8) (Fig. 2).

Based on their annotation information, similar exon/intron structures were observed in most of the SiAATs in the same subfamily, such as SiATLa1 and SIATLa5, SiCAT3 and SiCAT10, and SiATLb3 and SiATLb2. Variants were also found in some SiAATS of the same subfamily, such as SiAUX2 and SiAUX3, SIAAP11 and SIAAP18, and SIANT1 and SIANT2 (Fig. 3). The conserved motifs of SiAAT proteins predicted with MEME were highly consistent with the phylogenetic relationship and classification of SiAATs (Fig. 3; Additional file 2: Figure S2). Similar to their gene structures, the presence of the conserved motifs in different subfamilies of SiAAT proteins varied. For instance,Motif 1, 4, and 7 were widespread in the AAAP family, while Motif 9 existed in the APC family. Some motifs were unique to certain subfamilies, for example, AUX subfamily only possessed Motif 19 , and Motif 16 and 18 were only found in the ACT subfamily.

\section{Variations in gene structure, conserved domains and sequences of paralogous AAT genes promoted their functional differentiation}

The diversification of paralogous gene is one of the important sources of the functional differentiation of gene families [46-48]. The comparison on the TM regions of SiAATs found that significant variations in the number of TM regions occurred in the same duplicated gene groups, $60 \%$ of the duplicated gene groups (15/25) varied in the number of TM regions, which was $77 \%(10 / 13)$ and $42 \%(5 / 12)$ for the tandemly and for the segmentally duplicated gene groups, respectively (Fig. 1b; Table 2). 
Table 2

The extensive variations in gene structure, protein structure and sequence of paralogous $A A T$ genes produced by gene duplication in foxtail millet.

\begin{tabular}{|c|c|c|c|c|c|c|c|}
\hline \multirow{2}{*}{$\begin{array}{l}\text { Type of } \\
\text { duplication }\end{array}$} & \multirow[t]{2}{*}{ Subfamily } & \multirow{2}{*}{$\begin{array}{l}\text { No. of } \\
\text { duplicated } \\
\text { gene groups }\end{array}$} & \multicolumn{3}{|c|}{ Gene and protein structure variation } & \multirow{2}{*}{$\begin{array}{l}\text { Range of } \\
\mathrm{Ka} / \mathrm{Ks} \text { values }\end{array}$} & \multirow{2}{*}{$\begin{array}{l}\text { Duplicated } \\
\text { gene } \\
\text { groups }\end{array}$} \\
\hline & & & $\begin{array}{l}\text { No. of } \\
\text { TM } \\
\text { variation }\end{array}$ & $\begin{array}{l}\text { No. of } \\
\text { gene structure } \\
\text { variation }\end{array}$ & $\begin{array}{l}\text { No. of } \\
\text { conserved domain } \\
\text { variation }\end{array}$ & & \\
\hline \multirow{8}{*}{$\begin{array}{l}\text { Tandem } \\
\text { duplication }\end{array}$} & AAP & 4 & 3 & 3 & 2 & $0.12-0.62$ & TD1-TD4 \\
\hline & ATLb & 3 & 3 & 1 & 2 & $0.27-0.47$ & TD5-TD7 \\
\hline & GAT & 1 & 0 & 1 & 1 & 0.43 & TD8 \\
\hline & LHT & 2 & 2 & 2 & 0 & $0.10-0.39$ & TD9,TD10 \\
\hline & ACT & 1 & 0 & 1 & 1 & $0.18-0.27$ & TD11 \\
\hline & CAT & 1 & 1 & 1 & 0 & 0.97 & TD12 \\
\hline & PHS & 1 & 1 & 0 & 1 & $0.41-0.67$ & TD13 \\
\hline & Sum $^{a}$ & 13 & $10(77 \%)$ & $9(69 \%)$ & 7 (54\%) & $\begin{array}{l}0.10-0.97 \\
(0.28)\end{array}$ & \\
\hline \multirow{6}{*}{$\begin{array}{l}\text { Segmental } \\
\text { duplication }\end{array}$} & ATLa & 2 & 0 & 0 & 2 & $0.10-0.12$ & SD1,SD2 \\
\hline & ATLb & 3 & 2 & 2 & 2 & $0.12-0.40$ & SD3-SD5 \\
\hline & LHT & 2 & 2 & 1 & 0 & $0.12-0.37$ & SD6,SD7 \\
\hline & ACT & 1 & 0 & 1 & 0 & 0.10 & SD8 \\
\hline & CAT & 4 & 1 & 1 & 3 & $0.14-0.50$ & SD9-SD12 \\
\hline & Sum $^{a}$ & 12 & $5(42 \%)$ & $5(42 \%)$ & 7 (58\%) & $\begin{array}{l}0.10-0.50 \\
(0.21)\end{array}$ & \\
\hline
\end{tabular}

Multi-sequence alignment of SiAAP proteins revealed that their overall similarity was $59.65 \%$ with 11 conserved motifs, and their TM regions were highly correlated with the conserved motifs, both in length and amino acid composition (Fig. 4). Motif 1 and 14 both formed the TM 1 and TM 2 regions, TM 4 and part of TM 5 regions included Motif 4. TM regions of 3,6, 7, 8, 9 and 10 were with Motifs of 8, 6, 2, 7, 5, 13, respectively (Fig. 4). In addition, some conserved motifs were located in the non TM region, such as Motif 11 in the extra-membrane region and Motif 3 in the intra-membrane region (Fig. 4). It was worth noting that some SiAAPs had missing transmembrane regions due to incomplete conserved motifs, such as SiAAP6 and SiAAP9. Therefore, the number variation of TM regions was mainly determined by the presence of different conserved domains. Prediction of the secondary structure of SiAAT proteins found 14 a-helixes and $4 \eta$-helixes structures, with vast majority located in the TM regions to ensure the efficient and stable transmembrane transport of amino acids (Fig. 4).

In terms of gene structure, $56 \%(14 / 25)$ of duplicated gene groups had variation in the number of introns, which was $69 \%$ (9/13) of the tandemly ones and $42 \%$ of the segmentally ones (Table 2; Additional file 3: Figure S3). In addition, the variations in the conserved motifs were also observed in 14 duplicated gene groups, 7 for each of the two types of duplication (Table 2).

The $\mathrm{Ka} / \mathrm{Ks}$ values of all paralogous SIAAT gene pairs were less than 1 , ranged from 0.1 to 0.97 , which suggested that these genes were subject to different levels of purifying selection (Fig. 5). The tandemly duplicated gene pairs had higher Ka/Ks values than the segmental duplications, with the average Ka/Ks values of 0.28 and 0.21 , respectively (Table 2). The Ka/Ks values of tandemly duplicated genes of PHS subfamily ranged from 0.41 to 0.67 , while that of PHS subfamily only ranged from 0.18 to 0.27 . The Ka/Ks values of different subfamilies also showed different degrees of dispersion. For instance, that of SiAAPS ranged from 0.12 to 0.62 , while that of SiBATs (ACT subfamily) from 0.18 to 0.27 . This similar phenomenon was also observed on the Ka/Ks values of segmentally duplicated genes (Fig. 5). In general, significant variations in gene structure, conserved domains and sequences among the paralogous SiAAT gene groups might greatly promote the functional diversity of the AAT gene family in foxtail millet.

\section{Variations in expression levels of paralogous and orthologous SiAAT genes together drove the functional differentiation of AAT family in foxtail millet}

The pattern of expression of all paralogous $A A T$ genes were investigated using the public transcriptome data. Compared with the ancestral genes, according to their expression patterns, the newly duplicated genes could be classified into three main types of new-functionalization, sub-functionalization and nonfunctionalization, which were all observed in the tandemly duplicated gene groups with SiAAP8/9 (TD2) possessing new functions. SiAAP9 and SiAAP8 were highly expressed during grain development, and in leaves at the filling stage, respectively. SiAAP14/15/16/17(TD3) and SiCAT1/2 (TD12) showed subfunctionalization, as the expressions of SiAAP16/17 and SiCAT2 were down-regulated significantly. In addition, SiATLb12 and SiLHT3 losing their functions, as they were not expressed in any tissues (Fig. 6a). Though no new function was observed, similar functional differentiation were also observed in the segmentally duplicated gene groups, such as sub-functionalization of SiATLb1/6/10 (SD3), SiBAT1/SiBAT8 (SD8) and SiCAT3/10 (SD9), and nonfunctionalization of SiATLa1/5(SD1), SiLHT1/2 (SD6) and SiCAT4/11 (SD10) (Fig. 6b). 
There were 62, 51, 52 and 35 orthologous $A A T$ genes found in sorghum, rice, wheat and Arabidopsis, respectively, and their corresponding relationships with foxtail millet were investigated (Fig. 7; Additional file 6: Table S2). The wheat genome was much larger than other species, so it was not displayed in Fig. 7. The collinear relationship between different species was clearly distinguishable and showed good collinearity between chromosome 9 of foxtail millet and chromosome 1 of sorghum, chromosomes 3, 4, 5 of foxtail millet and chromosomes 8, 9, 10 of sorghum, respectively, etc. Moreover, the collinearity of the orthologous $A A T$ genes among different species was consistent with that of the whole genomes. The number of orthologous subfamilies $L H T s$ and $L A T S$ in millet and sorghum were significantly more than those in wheat and rice, which suggested that their expansion in millet and sorghum completed later than that in sorghum and wheat (Fig. 8; Additional file 6: Table S2). Compared with Arabidopsis, the AAP subfamily in grass species was significantly expanded. For instance, the orthologous of SIAAP2, SiAAP12 and SIAAP14 only could be found in grass species, which suggested that they might be produced after the differentiation of monocotyledon and dicotyledon.

The expression characteristics of those orthologous $A A T$ genes in root, stem, leaf, inflorescence/spike and grain of foxtail millet, sorghum, wheat, rice and Arabidopsis were compared using their intergrated transcriptome datasets. Owing to the heterohexaploid nature of wheat with almost three homologous copies for each gene, the average expression values of these copies were used. The expression patterns of SiAATs in foxtail millet and their orthologous in other species were generally conservative, especially in the grass species (Fig. 8). The correlation coefficients on the expression patterns of $A A T$ genes in gramineous species were $0.483,0.470$, and 0.481 between foxtail millet with sorghum, wheat and rice, respectively, while it was only 0.21 between foxtail millet and Arabidopsis. Several high-expressed AAT genes in foxtail millet were also highly conserved in other species, such as SiCAT3 and SiATLa5. In addition to the relatively conservative expression patterns of some important orthologous $A A T$ genes among different species, the expression patterns of some genes were differentiated, such as SiATLa6 and SiATLb6 (Fig. 8). Compared with the orthologous genes in Arabidopsis, SiAATs showed greater variation in expression patterns, and several genes changed their tissue specificities, such as SiATLb1 and SiAUX3.

\section{SiAATs showed abundant spatiotemporal expression pattems, and some of them were specifically expressed in developing grains}

The expression patterns of SiAATs were analyzed using the RNA-seq data of different tissues at mutiple growth stages collected from the public online database. The heat map was displayed with the normalized $\log _{2}(\mathrm{FPKM}+1)$ values (Fig. 9a). According to the expression patterns, the SiAATs were clustered into three groups. The 8 SiAAT genes in the first group included 7 AAAP family genes and one APC family gene with relatively high expression level, and were stably expressed in almost all tissues at different developmental stages. The 37 SiAAT genes in the second group were expressed in low abundance in most tissues, but expressed explicitly in some tissues, such as SiAAP9 and SiAAP7 were highly expressed in germinating seeds, while SiATLb3, SiAUX3 and SiAUX4 were relatively high expressed in panicle. The 49 SiAAT genes in the third group showed diverse spatiotemporal expression characteristics, such as SiATLa6 and SiATLa3 were highly expressed in the leaves at the seedling stage, but lower in the leaves at filling stage; SiAAP20 was specifically high-expressed in the stem, while SIAAP3, SiBAT7, SiCAT11, SiBAT2, SiLAT7 and SiLAT8 were expressed abundantly in leaf tissues including leaf sheath and mesophyll. In addition, some genes were highly expressed in two or more tissues, such as SIAAP1, SiAAP13 and SiAUX1 in stem and root; SIANT1, SiCAT3 and SIATLb1 were expressed abundantly in organs involved in the entire source-sink circulation including root, stem, leaf and grain. The expressions of the 20 selected SiAATs by qRT-PCR analysis were not completely consistent with that from the transcriptome data, but their expression characteristics and trends were similar, which verified the reliability of the transcriptome data (Fig. 9a; Additional file 4: Figure S4).

The transcriptome analysis of spike RNA-seq data showed that about half of SiAATs were expressed lowly during seed development, while the remaining ones were expressed at relatively high levels at different stages (Fig. 9b). SiAAP2, SiAAP9, SiATLa5, SiLAT5, SiATLb2 and SiBAT1 etC, were highly expressed in spikelets and grains throughout the grain development process (from S1 to S5). SiATLa6, SiAAP8, SiAAP20, SiAAP1, SiLAT6, SiCAT2 and SiCAT3, etc were highly-expressed at the early stage of grain development. SiGAT6 and SiProT1, etc were highly expressed at specific stages. In addition, the numbers of the highly expressed SiAATs during grain development in different subfamilies were counted, as 14 SiAAPS, 8 SiCATS, 5 SiLATs, 5 SiATLas and 3 SiAUXs, which accounted for $70 \%$ of total SiAAPs (20), 67\% of SiCATs (12), $63 \%$ of SiBATS (PHS, 8), 83\% of SiATLas (6) and 75\% of SiAUXs (4), respectively (Fig. 10).

There were significant differences in multiple grain quality traits between the two foxtail millet genotypes "JG21" and "YG1". Except for crude fat content, the basic amino acid contents of glutenin, cysteine, alanine, methionine, leucine, tryptophan and serine in "JG21" grain was significantly lower than "YG1" (Fig. 11b). The developing grains (including glumes) of "JG21" and "YG1" at filling stage in 2019 were separated for RNA extraction and RNA-sEq. Finally, 348 differentially expressed genes (DEGs) in the developing grains of these two genotypes were identified, of which 164 and 184 DEGs were down-regulated and up-regulated in "JG21", respectively. GO analysis showed that DEGs were mainly enriched in multiple terms such as responses to fungus or viruses, ribonuclease activity, stress response, and transmembrane transport of various substances (Fig. 11a). Interestingly, 9 AAT genes were enriched into four terms related to amino acid transport, including 5 SiAAPS, 1 SiANT, 1 SiATLb, 1 SiAUX and 1 SiBAT (Fig. 11c). The differential expression of these SiAATs suggested that these genes might directly affect the formation of grain quality.

\section{Some SiAATs actively responded to various abiotic stresses}

The qRT-PCR results of JG21 and the transcriptome data of the foxtail millet cultivars of Yugu1 and Yugu2 showed a relatively consistent trend of a similar response of SiAAT genes to abiotic stress (Fig. 12a). The qRT-PCR analysis of 12 SiAATs in foxtail millet seedlings (15 days after sowing) to simulated drought $(20 \%$ PEG $6000,1 \mathrm{~h}$ and $5 \mathrm{~h}$ ) and salt $(200 \mathrm{mM} \mathrm{NaCl}, 1 \mathrm{~h}$ and $5 \mathrm{~h}$ ) revealed that various subfamilies of SiAATs responded differently to abiotic stresses (Fig. 12b). Seven SiAAT genes were up-regulated more than 5 times after drought or salt stress, of which SiANT1 was up-regulated by about 60 times at $5 \mathrm{~h}$ after drought stress. SIANT1, SiCAT10 and SiATLa1 were mainly induced by drought stress, and their expression all reached the highest levels at $5 \mathrm{~h}$ after drought treatment. Except for SIAUX2 and SiATLb5, the other 7 SiAAT genes reached their highest expression levels at the late stage of salt stress (5 $\mathrm{h}$ ). SiLHT12, SiATLb1 and SiLAT3 were specifically and strongly induced by long-time salt stress, while SiANT1, SiATLa1 and SiATLb5 had similar expression patterns under both drought and salt stresses. 
The response of different SiAATs to abiotic stress also showed temporal specificity. SiAAP3 was up-regulated under $1 \mathrm{~h}$ drought stress, while it was upregulated at $5 \mathrm{~h}$ under salt stress. SiLHT12 was down-regulated under $1 \mathrm{~h}$ drought and salt stresses, but then was strongly up-regulated at $5 \mathrm{~h}$ (Fig. $12 \mathrm{~b}$ ). SiANT1 was continually up-regulated with the increase of drought stress, and was down-regulated under $5 \mathrm{~h}$ salt stress. SiATLb5 and SiATLa1 responded at the early and late stages of drought and salt stress, respectively. Further, some SiAATS showed a sustainable response to abiotic stresses; for example, both SiATLb2 and SiATLa1 were continually up-regulated under both salt and drought stresses. These genes might enhance the adaptability of foxtail millet to abiotic stresses through their active responsiveness.

Table 3

Functional prediction of $A A T$ genes in foxtail millet based on homology relationship, expression pattern and previous studies.

\begin{tabular}{|c|c|c|c|c|}
\hline Subfamilies & $\begin{array}{l}\text { AATs in foxtail } \\
\text { millet }\end{array}$ & $\begin{array}{l}\text { expression } \\
\text { organization }^{a}\end{array}$ & Possible biological processes & Previous studies \\
\hline AAP & $\begin{array}{l}\text { SiAAP7; } \\
\text { SiAAP1,5,13; } \\
\text { SiAAP2,10,9; } \\
\text { SiAAP3,8,12,14 } \\
\text { SiAAP4,15,19,20 }\end{array}$ & $\begin{array}{l}\text { GS; } \\
\text { R,ST; } \\
\text { GS,SP,G } \\
\text { LS,LV, G } \\
\text { ST,LS,LV }\end{array}$ & $\begin{array}{l}\text { The intake of amino acid in } \\
\text { endosperm and embryo } \\
\text { development; } \\
\text { Amino acid absorption in root and } \\
\text { transport in stems; } \\
\text { Play a role in early embryo } \\
\text { development and formation of floral } \\
\text { organs and grain quality; } \\
\text { Participate in the transport and } \\
\text { distribution of amino acids in leaf } \\
\text { and grain formation; } \\
\text { Participate in the transfer and } \\
\text { distribution of amino acids from } \\
\text { stems to leaf }\end{array}$ & $\begin{array}{l}\text { AtAAP8, }{ }^{[58,61]} \text {; } \\
\text { AtAAP7, }{ }^{[19] ;} \\
\text { AtAAP8, } 1 \text {,VfAAP1,OsAAP6 }{ }^{[62,4,7] ;} \\
\text { HvAAP3,AtAAP7 }{ }^{[25]} \text {;AtAAP2,3,6,OsAAP8, 15; HvAAP2, } 6^{[20,21,8,12]}\end{array}$ \\
\hline GAT & $\begin{array}{l}\text { SiGAT3; } \\
\text { SiGAT6 }\end{array}$ & $\begin{array}{l}\mathrm{LS}, \mathrm{LV}, \mathrm{L}, \mathrm{SP} \\
\mathrm{R}, \mathrm{ST}, \mathrm{LS}, \mathrm{SP}\end{array}$ & $\begin{array}{l}\text { Maintain cell ion balance under } \\
\text { normal conditions; } \\
\text { Amino acid absorption in root and } \\
\text { long-distance transportation of } \\
\text { GABA }\end{array}$ & AtGAT $^{[44]}$ \\
\hline Prot & SiProT1 & ST,L,GS & $\begin{array}{l}\text { Participate in the long-distance } \\
\text { transport of proline and regulate the } \\
\text { distribution of proline in leaf }\end{array}$ & AtProT1,AtProT3 $3^{[5,38]}$ \\
\hline LHT & $\begin{array}{l}\text { SiLHT11 } \\
\text { SiLHT2,5,10 } \\
\text { SiLHT9 }\end{array}$ & $\begin{array}{l}\mathrm{R} ; \\
\mathrm{L} ; \\
\mathrm{LS}, \mathrm{ST}, \mathrm{R}\end{array}$ & $\begin{array}{l}\text { Amino acid uptake in root; } \\
\text { Transport and distribution of amino } \\
\text { acids in leaf; } \\
\text { Long-distance transportation of } \\
\text { amino acids from root to leaf }\end{array}$ & AtLHT1,9 $[28,63]$ \\
\hline AUX & $\begin{array}{l}\text { SiAUX1; } \\
\text { SiAUX2,3,4; }\end{array}$ & $\begin{array}{l}\mathrm{ST}, \mathrm{R} ; \\
\mathrm{SP} ; \mathrm{G}\end{array}$ & $\begin{array}{l}\text { Participate in the geotropism of root } \\
\text { and the transport of auxin in the } \\
\text { stem; } \\
\text { Participate in spikelet development } \\
\text { and grain formation }\end{array}$ & $\begin{array}{l}\text { AtAUX1 }{ }^{[32-34]} ; \\
\text { OsAUX1,StAUX }\end{array}$ \\
\hline ANT & $\begin{array}{l}\text { SiANT1; } \\
\text { SiANT2 }\end{array}$ & $\begin{array}{l}\mathrm{ST}, \mathrm{R}, \mathrm{LS}, \mathrm{LV} \\
\mathrm{SP}, \mathrm{G}, \mathrm{GS}\end{array}$ & $\begin{array}{l}\text { Participate in long-distance } \\
\text { transportation of amino acids; } \\
\text { Participate in seed germination, } \\
\text { spike differentiation and grain } \\
\text { formation }\end{array}$ & AtANT $1^{[31]}$ \\
\hline ATLa & $\begin{array}{l}\text { SiATLa1; } \\
\text { SiATLa3,4,5; } \\
\text { SiATLa6 }\end{array}$ & $\begin{array}{l}\text { GS,SE; } \\
\text { ST,LS,LV,R,SP; } \\
\text { L,SP }\end{array}$ & $\begin{array}{l}\text { Participate in seed germination; } \\
\text { Participate in long-distance } \\
\text { transportation of amino acids; } \\
\text { Regulate the distribution of amino } \\
\text { acids in leaf and Participate in spike } \\
\text { differentiation }\end{array}$ & NA \\
\hline ATLb & $\begin{array}{l}\text { SiATLb3,6,10,13; } \\
\text { SiATLb1; } \\
\text { SiATLb7; } \\
\text { SiATLb8; }\end{array}$ & $\begin{array}{l}\text { GS,SE,SP,G; } \\
\text { R,ST,LV; } \\
\text { GS; } \\
\text { ST,LS,LV }\end{array}$ & $\begin{array}{l}\text { Participate in seed germination, } \\
\text { spike differentiation and grain } \\
\text { formation; } \\
\text { Long-distance transportation of } \\
\text { amino acids from root to leaf; } \\
\text { Participate in seed germination; } \\
\text { Long-distance transportation of } \\
\text { amino acids from stem to leaf; }\end{array}$ & NA \\
\hline
\end{tabular}


Table 3

continued.

\begin{tabular}{|c|c|c|c|c|}
\hline Subfamilies & $\begin{array}{l}\text { AATs in foxtail } \\
\text { millet }\end{array}$ & $\begin{array}{l}\text { expression } \\
\text { organization } 1\end{array}$ & Possible biological processes & $\begin{array}{l}\text { Previous } \\
\text { studies }\end{array}$ \\
\hline \multirow[t]{4}{*}{ CAT } & SiCAT10 & GS & Participate in seed germination; & \multirow[t]{4}{*}{ NA } \\
\hline & SiCAT3,5,6,8,9 & $\mathrm{GS}, \mathrm{ST}, \mathrm{R}, \mathrm{L}$ & Participate in long-distance transportation of amino acids; & \\
\hline & SiCAT7 & SP & Specific influence on spike differentiation; & \\
\hline & SiCAT11,12 & $\mathrm{SP}, \mathrm{L}$ & $\begin{array}{l}\text { Participate in spikelet formation and amino acid transport and distribution } \\
\text { in leaves }\end{array}$ & \\
\hline ACT & SiBAT1,2,7 & ST,L,SP & $\begin{array}{l}\text { Participate in spikelet formation and long-distance transportation of } \\
\text { amino acids }\end{array}$ & NA \\
\hline \multirow[t]{2}{*}{ PHS } & SiLAT1,2,5 & GS,SP,G; & Participate in seed germination, spike differentiation and grain formation; & \multirow[t]{2}{*}{ NA } \\
\hline & SiLAT3 & GS,LS,LV,ST,R & $\begin{array}{l}\text { Participate in spikelet formation and long-distance transportation of } \\
\text { amino acids }\end{array}$ & \\
\hline TTP & SiTTP1 & SE,SP & Affect seedling growth and spikelet differentiation & NA \\
\hline
\end{tabular}

\section{Discussion}

\section{A large number of tandem and segmental duplication events were the direct cause ofAAT gene family expansion in foxtail millet}

Amino acid transporters (AATs) play indispensable roles in plant growth by transporting and distributing different types of amino acids, and therefore are considered as important targets for crop improvement [15]. Here, a total of 94 AAT genes were characterized in foxtail millet, which were divided into 12 subfamilies and was consistent with the previous reports in other plants (Table 1). Different subfamilies of AATs in monocotyledons and dicotyledons were corresponding, thus confirming that they were formed before the differentiation in monocotyledons and dicotyledons (Fig. 2). The number of SiAATs in foxtail millet was less than those in hexaploid wheat (294) and maize (107) [13], but more than that in sorghum (77), rice (85) [12], and Brachypodium [13], and much higher than that in Arabidopsis (63) [1] and potato (72) [10]. Earlier studies indicated that the plant $A A T$ gene family originated from the prokaryotic genome through horizontal gene transfer and subsequent duplication events [49]. There were 47, 16, 22 AAT genes associated with duplication events in rice, Arabidopsis and potato, accounting for $55.29 \%$ (47/85) [12], 25.40\% (16/63) [10] and 30.55\% (22/72) [10], respectively. As observed in this study, 58 of the 94 (62\%) SiAATs were duplicated in foxtail millet, including 36 and 25 tandemly and segmentally duplicated genes, respectively. All these further verified the influence of gene duplication of the AAT family expansion in monocots. Furthermore, obvious imbalances in the expansion of different $A A T$ subfamilies in monocots and eudicots were found, as AAP, ATLa/b, ACT and CAT subfamilies expanded greatly in monocots, while more ProT genes in eudicots (Table 2; Fig. 13). These increased AATs may play important roles in specific functions, and promoted further differentiation of monocots and eudicots.

\section{The multi-levels variations of paralogousAAT genes generated from gene duplication promoted the functional diversity of AAT family in foxtail millet}

Gene duplication and subsequent functional differentiation of new genes promoted the expansion and new-functionalization of important gene families, thereby enabling plants to better adapt to the environment [46]. Variations in the sequence, structure and expressions of genes were the three main sources of functional differentiation [13]. Base mutation was the most direct evidence of sequence variation. In general, the Ka/Ks values of all duplicate groups except for TD12 were far less than 1, indicating that most of the paralogous $A A T$ genes undergone purifications in sequence variation to ensure the stability of key biological functions (Fig. 5). The Ka/Ks values of different AAT subfamilies showed different median and dispersion, such as AAP, PHS and CAT subfamilies had higher dispersion, which indicated that these subfamilies may have the opportunity to produce new features. However, the Ka/Ks values of ACT subfamily, both tandemly and segmentally duplicated gene pairs, showed smaller dispersion, suggesting that their functions might be more conservative. In addition, tandemly duplicated gene pairs showed higher $\mathrm{Ka} / \mathrm{Ks}$ values than segmentally duplicated gene pairs, suggesting they were under stronger selection pressure, which confirmed that tandem duplication played more crucial roles in functional diversity of foxtail millet $A A T$ genes. Similar results were also found in the study of wheat $A A T$ family genes [13]. These results confirmed that the sequence variations of paralogous genes in different AAT subfamilies of foxtail millet increased the diversity of $A A T$ genes.

Previous studies have shown that variations in gene structure are also important markers of gene family evolution [48, 49]. In this study, 56\% (14/25) of the paralogous gene groups had significant changes in the number of introns, resulted in 9 and 5 groups of tandem and segmental duplications, respectively, accounting for $69 \%$ and $42 \%$ of the total (Table 2; Additional file 3: Figure S3). The variations in the number of TM regions were found in $77 \%$ (10/13) and $42 \%$ $(5 / 12)$ of the tandemly and segmentally duplicated gene groups, respectively, which were caused by the deletion of conserved domains, such as SiAAP5/6/7 (TD1), SiAAP8/9 (TD2) (Fig. 1b; Fig. 4). There were significant variations in gene structure and the number of TM regions which was observed in 56\% (14/25) and $60 \%(15 / 25)$ of the two duplicated gene groups, confirming that the structural variation of paralogous genes was one of the important sources of the functional categorization of the $A A T$ family genes in foxtail millet.

Changes in expressions of paralogous genes are another key sources of functional categorization of important gene family [49]. Sub-functionalization and new-functionalization of new duplicated genes directly lead to significant differences in expression level and spatiotemporal expression patterns, compared to their ancestor genes [48]. As observed in rice [12], soybean [11] and potato [10], the changes of the three expression types of paralogous genes were also observed in this study. For instance, SiAAP8/9 (TD2) and SiAAP19/20 (TD4) were specifically expressed in different tissues, (Fig. 6), confirming that the 
expression changes of paralogous genes in AAT family were common in higher plants. Interestingly, the paralogous genes with new functions were only found in tandemly duplicated gene groups, suggesting that tandem duplication has a greater contribution to the evolution of the AAT gene family in foxtail millet.

\section{The changes of orthologousAATgenes in foxtail millet play important roles in differentiation of new functions}

Along with the separation of species and the influence of natural selection, though the orthologous genes of different species are largely conserved, the variations in their regulatory elements, epigenetic markers, etc will cause changes in the pattern of expression of orthologous genes in various species, resulting in their functional differentiation [50-54]. For some $\mathrm{C}_{4}$ genes in $\mathrm{C}_{4}$ species, such as $P E P C, M D H$ and $P P D K$, their $\mathrm{C}_{4}$ functions were directly obtained by the differences in the subcellular localization and expression level of orthologous genes [55]. The completion of genome sequencing allowed us to quickly identify the orthologous genes of different plants at genome level (Fig. 7). There were 62, 51, 52, 35 orthologous of the SiAATs identified in sorghum, rice, wheat and Arabidopsis, which were consistent with the genetic distance and differentiation time of these species [56]. The AAP subfamily of grass species expanded significantly after the differentiation of monocots and eudicots, which was consistent with previous studies [12, 13]. Within the grass family, the LHT and LAT subfamilies expanded significantly in foxtail millet and sorghum, which may have improved their adaptability to the environment. Most SiAATs and their orthologous in other species had similar expression patterns, especially for those of grass family with high correlation coefficients ranged from 0.470 to 0.483 (Fig. 8). Compared with their orthologous in Arabidopsis, the tissue expression characteristics of some SiAATs had changed, which may be related to the long genetic distance and complete differentiation between foxtail millet and Arabidopsis.

\section{Many importantAAT genes were expressed conservatively in multiple species to retain essential biological functions}

Spatiotemporal expression analysis of important gene family members will help to predict their putative functions [57]. Some SiAATs, such as SiProT1 and SiATLa5, were abundantly expressed during the whole period of growth, which were essential for the growth and development of foxtail millet (Fig. 9). Some SiAATs were abundantly expressed in specific organs at certain developmental stages, such as SiAAP9, SiAAP7 and SiAAP2 in germinating seeds, SiAAP1 and SIAUX1 in stems and roots, and SiLAT7 and SiLAT8 in leaf tissues, while, SiAAT6 was highly expressed only in leaves at seedling and heading stages, but low at filling stage. The different spatiotemporal expression patterns of these genes suggested that they might have specific functions.

The functions of some $A A T$ genes have been extensively studied in the model species, such as $A t A A P 1$ for seed germination and amino acid intake in root [9, 14, 58]. It was found that SIAAP7 and SIAAP2, OSAAP1 [26] ppossessed similar functions; AtAAP3 in the uptake of amino acids in xylem, and SiAAP4 highly expressed in stems and veins [21]; StAAP1 was expressed in multiple organs of source-sink transport in potato [24, 10]; AtLHT1 located in root epidermis and mesophyll cells in Arabidopsis, which was primarily responsible for the amino acid uptake in soil [28, 29]; AtAUX1, AtLAX3 and OsAUX1 related to the gravitropism of root and lateral root formation [12, 32, 33]. As similar expression patterns were also observed for orthologous genes, which maintain the important process of plant growth and development. Therefore, we preliminary predicted their functions in foxtail millet based on these previous studies, the phylogenetic relationships and the spatiotemporal expression characteristics of SiAATs in foxtail millet (Table 3).

\section{MultipleAAT genes may contribute directly to grain quality traits in foxtail millet}

The $A A P$ genes are closed related to grain development and quality formation in many species. AtAAP1 in Arabidopsis and OsAAP6 in rice are directly related to grain protein content [7, 14], multiple $A A T$ genes were located in or near the QTL regions associated with quality traits in wheat by genome-wide association analysis (GWAS) [27]. The RNA-seq analysis of the foxtail millet grains at development stages revealed that 70\% AAPS (14/20), 67\% CATs (8/12), 63\% BATs (5/8), 83\% ATLa (5/6) and 75\% AUXs (3/4) were highly expressed during grain development, including SiAAP9 and SiAAP8. The orthologous genes of OsAAP6, suggested that those SiAATs played a major role in the development and quality formation of foxtail millet grain (Fig. 10). The DEGs between the two cultivars were enriched into four GO terms related to amino acid transport (Fig. 11a). Nine SiAATs, including SiAAP8, belonging to AAP, ANT, ATLa, AUX and PHS subfamilies, were differentially expressed in the developing grains of the two genotypes, which further confirmed that they might directly affect the grain quality traits (Fig. 11c). All these suggested that it might be feasible to improve the quality traits by manipulating the expressions of those important SiAAT genes in foxtail millet, which also provide a base for further analysis of their function in quality formation in foxtail millet.

\section{SiAATsenhanced the adaptability of foxtail millet to abiotic stresses}

Drought and salt seriously affect crop yield and grain quality, and are the most important abiotic stresses in agricultural production. In Arabidopsis and other species, it has been confirmed that $A A T$ genes in higher plants regulate the balance of osmotic potential to resist the impact of stress on plant growth, mainly through the transport of a variety of stress-response compounds and compatible solutes, such as betaine, GABA, and proline [40-44]. AtAAP4 and AtAAP6 were down-regulated under salt stress [42], and OSAAP4, OsAAP8 and OsBAT4 were down-regulated both under salt and drought stresses [12]. AtProT2 [42], MCAAT1 [59] and HVProT [60] were greatly triggered by salt stress. In this study, active responsiveness to simulated drought and salt stresses were observed in 12 SiAAT genes investigated, and various response patterns to different stresses were found in different subfamily members (Fig. 12). It should be noted that SiAATS located on vacuole membranes such as SiANT1 and SiALTb2 responded stronger to these abiotic stress, which may relate to their roles in rapid adjustment of cell osmotic potential to maintain cell water balance. These genes could be used as key candidate genes to improve resistance and tolerance under abiotic stresses.

\section{Conclusions}

In this study, 94 AAT genes in foxtail millet were systematically identified and characterize and were divided into 12 subfamilies. The paralogous genes generated by tandem duplication and segmental duplication promoted $A A T$ gene family expansion. The variations in sequence, structure and the pattern of expression of tandemly duplicated paralogous $A A T$ genes had significant impacts on their functional differentiation. The functional differentiation of SiAATS was the result of the unequal expansion and differentiation of paralogous genes in different subfamilies. The transcriptome analysis revealed the 
contributions of some $A A T$ genes in the formation of millet quality. Multiple SiAAT genes actively responded to drought and salt stresses as revealed by qRTPCR. The possible functions of the SIAAT family members were predicted. These findings might provide valuable information for further functional analysis of $A A T$ genes and their applications in the improvement of foxtail millet.

\section{Methods}

\section{Genome-wide scan and identification of AAT genes in foxtail millet}

The latest foxtail millet genomic and protein sequences were obtained from the ensemble plants database (http://plants.ensembl.org/info/website/ftp/index.html). First, the AAT proteins identified in Arabidopsis, rice and wheat were aligned with the millet protein sequences by BLASTP program. Second, the conserved domains of putative AAT proteins were checked using the Hidden Markov Model (HMM) profiles of the AAT domain (PF01490 and PF00324) from the Pfam database (http://pfam.xfam.org/), with a HMMER tool [64]. Third, the existence and integrity of the conserved domains in candidate proteins were further analyzed through conserved domain database (CDD) (https://www.ncbi.nlm.nih.gov/cdd) and InterPro database (http://www.ebi.ac.uk/interpro/scan.html). Finally, the $A A T$ gene family members in foxtail millet were identified after removing those containing incomplete conservative domains and redundant sequences.

Based on the chromosome position and phylogenetic relationship, these putative $A A T$ genes in foxtail millet were systematically named, and their detailed information was counted, including cDNA length, coding sequence length, gene structure and protein sequence feature. Their gene structures and biochemical parameters were determined by GSDS (http://gsds.cbi.pku.edu.cn/) and Computer pl/Mw tool (https://web.expasy.org/compute_pi/), respectively [65]. As complete membrane proteins, their transmembrane (TM) regions and subcellular localization were predicted by TMHMM server 2.0

(http://www.cbs.dtu.dk/services/TMHMM/) and LocTree 3 (https://www.rostlab.org/services/loctree3/), respectively [66].

Chromosomal mapping, duplication and selective pressure analysis

The locations of SiAAT genes on the chromosomes were obtained from the ensemble plants database. The gene duplication was determined by MCScanX, and manual screening was performed according to Wang et al. [67, 68]. The distributions of $A A T$ genes and their duplicated paralogous on chromosomes were ploted with Btools software [69]. The ratios (Ka/Ks) of non-synonymous (Ka) and synonymous (Ks) of these paralogous $A A T$ genes were analyzed using KaKs_Calculator 2.0 and displayed by an R package ggplot2 [70].

Phylogenetic and conserved motifs analysis

Multi-sequence alignment of the AAT protein sequences identified in Arabidopsis, potato, rice and foxtail millet was conducted with ClustalW, and phylogenetic tree was created using the maximum likelihood (ML) method, JTT model and a bootstrap of 1000 using MEGA 6.06 [71]. The phylogenetic tree was displayed with iTOL v3 (http://itol.embl.de/\#).

The conserved motifs were determined using Motif-based sequence analysis (MEME) program (http://meme-suite.org) with the parameters of motif width of 6-200, the maximum motif number of 20 and any number of repeating motif sites [72]. TM regions and the possible protein secondary structures obtained from Protein Data Bank (PDB) database (https://www.rcsb.org/) were annotated and displayed by ESPript 3.0 [73].

\section{Identification of the orthologous AAT genes in sorghum, wheat, rice and Arabidopsis, and their expression analysis}

The orthologous $A A T$ genes in sorghum, wheat, rice and Arabidopsis were identified as those in foxtail millet, and by OrthFinder2 [74]. Their expression data in the main organs of root, stem, leaf, inflorescence/spike, and grain was obtained from the public transcriptome databases, respectively (wheat, http://www.wheat-expression.com; rice, http://expression.ic4r.org; sorghum, http://structuralbiology.cau.edu.cn/sorghum/; Arabidopsis, https://www.arabidopsis.org). Then, the conservation of the expression patterns of $A A T$ orthologous genes among those species was investigated through the tissue-to-tissue and gene-to-gene correlation analysis, using the software of SPSS 19.0 (SPSS, Inc., Chicago, IL, USA).

To study the spatiotemporal expression patterns of SiAATs in various tissues and grain development, a merged transcriptome dataset of foxtail millet was constructed with the RNA-seq reads downloaded from Multi-omics Database for Setaria italica (MDSi, http://foxtail-millet.biocloud.net/home), including 13 tissues at the seedling, heading and filling stages, and 2 spikelets and 5 grains. The FPKM (Fragments Per Kilobase per Million) values were calculated using an $\mathrm{R}$ package Edge $\mathrm{R}$ [75]. The heat maps of $\log _{2}(\mathrm{FPKM}+1)$ values were drawn by TBtools [69].

Quality traits measurement and transcriptome analysis of two foxtail millet cultivars

Two foxtail millet cultivars "JG21" and "YG1" used in this experiment were widely grown in northern China and provided by Mr Junjie Wang of Center for agricultural genetic resources research, shanxi agricultural university. They were grown in the experimental farm of the Institute of Water Saving Agriculture in Arid Areas of China, Northwest A\&F University, Yangling, Shaanxi, China $\left(34^{\circ} 7^{\prime} \mathrm{N}, 108^{\circ} 4^{\prime} \mathrm{E}\right)$ for two cropping seasons (May to September of $2017-2018$ and 2018-2019), followed the local management practices. After harvest, the mature seeds were taken for measuring quality traits, including glutenin content, crude fiber content, crude fat content, and the contents of 18 essential amino acids of methionine, cysteine, phenylalanine, alanine, glycine, glutamic acid, arginine, lysine, tyrosine, leucine, proline, tryptophan, Serine, threonine, aspartic acid, valine, isoleucine, histidine, with a near-infrared analyzer DA7250 (Perten, Sweden).

The developing grains (include glumes) of "JG21" and "YG1" were separated at filling stage in 2019, and the total RNA was isolated using a Quick RNA Isolation kit (Takara Corporation, Dalian, China) [76], RNA purity and integrity was confirmed by an RNA Nano 6000 Assay Kit and the Bioanalyzer 2100 system (Agilent Technologies, CA, USA). The RNA-seq library construction and sequencing were performed with the 150 paired-end sequencing on Illumina HiSeq platform following the standard methods by Novogene Life Sciences Pvt. Ltd., Beijing, China. After filtered the raw data, Clean reads were then mapped to the reference genome using Hisat2 [77]. Differentially expressed genes (DEGs) between "JG21" and "YG1" were identified using the R package DESeq [78], 
and the FPKM values were also calculated [79]. The KEGG (Kyoto Encyclopedia of Genes and Genomes and GO (Gene Ontology, http://geneontology.org/), http://www.genome.jp/kegg) annotation of DEGs were further performed with GOseq and KOBAS software, respectively [80].

\section{The expressions of some AAT genes in response to abiotic stresses}

To verify the reliability of the transcriptome data from RNA-seq, the flag leaves, peduncles, roots, developing grains (include glumes) of "JG21" and developing grains (include glumes) of "YG1" were collected at the filling stage in 2019 for RNA extraction. For abiotic stresses, the raw read data of two datasets (SRA062640 and PRJNA545871) of foxtail millet under drought stress and salt stress were sourced from the NCBI SRA database to analyze the response of SiAATs to abiotic stresses [81, 82]. The detailed analysis process was described above.

The seedlings, 15 days after sowing (two leaves with a terminal bud) were exposed to $20 \%$ polyethylene glycol (PEG 6000$)$ solution and NaCl solution $(200 \mathrm{mM})$ for $1 \mathrm{~h}$ and $5 \mathrm{~h}$ to mimic salt and drought stress, respectively, and all seedling plants were then collected for RNA isolation. PrimeScriptTM II $1 \mathrm{st}$ Strand cDNA Synthesis was used to synthesize the first strand of cDNA and Quantitative Real-time PCR (qRT-PCR) was conducted by SuperReal PreMix Color kit (SYBR Green) with the primers listed in Table 3 and Additional file 7: Table S3, with the SiAct-7 gene as an internal reference. Each sample contained three biological replicates, and the $2^{-\triangle \Delta C T}$ method was used for the statistical analysis.

\section{Abbreviations}

AAT: Amino acid transporter; APC: Amino acid-polyamine-choline; AAAP: Amino acid/auxin permease; ACT: Amino acid/choline transporter; CAT: Cationic amino acid transporter; PHS: Polyamine $\mathrm{H}^{+}$-symporter; TTP: Tyrosine-specific transporter; $\mathrm{LHT}$ : Lysine and histidine transporter; GAT: $\mathrm{Y}^{-}$aminobutyric acid transporter; ProT: Proline transporter; AAP: Amino acid permease; AUX: Auxin transporter; ANT: Aromatic and neutral amino acid transporter; GABA: $\gamma$ aminobutyric acid; QTL: Quantitative trait locus; pl : isoelectric point; Mw: Molecular weight; TM: Transmembrane; qRT-PCR: Quantitative Real-Time PCR; GPC: Grain protein content

\section{Declarations}

\section{Acknowledgements}

Special thanks to Mr Junjie Wang (Center for agricultural genetic resources research, shanxi agricultural university) for providing the foxtail millet materials used for experiments.

\section{Authors' contributions}

YY and YC performed the experiment and wrote the paper; JL collected the previous studies; JZ designed primers and performed the qRT-PCR; ZZ and CC performed transcriptome sequencing and data analysis; QL carried out quality trait investigation; AA checked the language specification of manuscript; YGH and LC designed the experiment and reviewed the paper. All authors read the final version of the manuscript.

\section{Funding}

This research was funded by the National Natural Science Foundation of China (31671695 and 31501307) and the China 111 Project of the Ministry of Education of China (B12007).

\section{Availability of data and materials}

The RNA-seq raw data of two foxtail millet cultivars "YG1" and "JG21" was submitted in the NCBI Sequence Read Archive (SRA) with the accession number PRJNA673778. The datasets supporting the conclusions of this article are included within the article and its additional files.

\section{Ethics approval and consent to participate}

Not applicable.

\section{Consent for publication}

Not applicable.

\section{Competing interests}

The authors declare that they have no competing interests.

\section{References}

1. Rentsch D, Schmidt S, Tegeder M. Transporters for uptake and allocation of organic nitrogen compounds in plants. FEBS Lett. 2007;581(12):2281-89. Doi:10.1016/j.febslet.2007.04.013.

2. Williams LE, Miller A. Transporters responsible for the uptake and partitioning of Nitrogenous Solutes. Annu Rev Plant Physiol Plant Mol Biol. 2001;52(1):659-88. Doi:10.1146/annurev.arplant.52.1.659.

3. Williams LE, Bick JA, Neelam A, Weston KN, Hall JL. Biochemical and molecular characterization of sucrose and amino acid carriers in Ricinus communis. J Exp Bot. 1996;47(Special Issue):1211-16. Doi:10.1093/jxb/47.Special_Issue.1211. 
4. Miranda M, Borisjuk L, Tewes A, Heim U, Sauer N, Wobus U, Weber H. Amino acid permeases in developing seeds of Vicia faba L.: expression precedes storage protein synthesis and is regulated by amino acid supply. Plant J. 2001;28(1):61-71. Doi:10.1046/j.1365-313X.2001.01129.X.

5. Ortiz-Lopez A, Chang H, Bush DR. Amino acid transporters in plants. Biochim Biophys Acta. 2000;1465(1-2):275-80. Doi:10.1016/s0005-2736(00)001449.

6. Rolletschek H, Hosein F, Miranda M, Heim U, Gotz KP, Schlereth A, Borisjuk L, Saalbach I, Wobus U, Weber H. Ectopic expression of an amino acid transporter (VfAAP1) in seeds of Vicia narbonensis and pea increases storage proteins. Plant Physiol. 2005;137(4):1236-49. Doi:10.1104/pp.104.056523.

7. Peng B, Kong HL, Li YB, Wang LQ, Zhong M, Sun L, Gao GJ, Zhang QL, Luo LJ, Wang GW, et al. OsAAP6 functions as an important regulator of grain protein content and nutritional quality in rice. Nat Commun. 2014;5(1):4847. Doi:10.1038/ncomms5847.

8. Hunt E, Gattolin S, Newbury HJ, Bale JS, Tseng HM, Barrett DA, Pritehard J. A mutation in amino acid permease AAP6 reduces the amino acid content of the Arabidopsis sieve elements but leaves aphid herbivores unaffected. J Exp Bot. 2012;61(1):55-64. Doi:10.1093/jxb/erp274.

9. Singh BK. Plant Amino Acids: Biochemistry and Biotechnology. New York: Marcel Dekker; 1999. pp. 227-47.

10. Ma H, Cao X, Shi S, Li S, Gao J, Ma Y, Zhao Q, Chen Q. Genome-wide survey and expression analysis of the amino acid transporter superfamily in potato (Solanum tuberosum L.). Plant Physiol Biochem. 2016;107:164-77. Doi:10.1016/j.plaphy.2016.06.007.

11. Cheng L, Yuan HY, Ren R, Zhao SQ, Han YP, Zhou QY, Ke DX, Wang YX, Wang L. Genome-wide identification, classification, and expression analysis of amino acid transporter gene family in glycine max. Front Plant Sci. 2016;7:515. Doi:10.3389/fpls.2016.00515.

12. Zhao H, Ma H, Yu L, Wang X, Zhao J. Genome-wide survey and expression analysis of amino acid transporter gene family in rice (Oryza sativa L.). PLoS ONE. 2012;7:e49210. Doi:10.1371/journal.pone.0049210.

13. Tian R, Yang Y, Chen M. Genome-wide survey of the amino acid transporter gene family in wheat (Triticum aestivum L.): Identification, expression analysis and response to abiotic stress. Int J Biol Macromol. 2020;162. Doi:10.1016/j.ijbiomac.2020.07.302.

14. Sanders A, Collier R, Trethewy A, Gould G, Sieker R, Tegeder M. AAP1 regulates import of amino acids into developing Arabidopsis embryos. Plant J. 2009;59(4):540-52. Doi:10.1111/j.1365-313X.2009.03890.x.

15. Okumoto S, Pilot G. Amino acid export in plants: a missing link in nitrogen cycling. Mol Plant. 2011;4(3):453-63. Doi:10.1093/mp/ssr003.

16. Tegeder M. Transporters for amino acids in plant cells: some functions and many unknowns. Curr Opin Plant Biol. 2012;15(3):315-21. Doi:10.1016/j.pbi.2012.02.001.

17. Su YH, Frommer WB, Ludewig U. Molecular and functional characterization of a family of amino acid transporters from Arabidopsis. Plant Physiol. 2004;136(2):3104-13. Doi:10.1104/pp.104.045278.

18. Okumoto S, Schmidt R, Tegeder M, Fischer WN, Rentsch D, Frommer WB, Koch W. High affinity amino acid transporters specifically expressed in xylem parenchyma and developing seeds of Arabidopsis. J Biol Chem. 2002;277(47):45338-46. Doi:10.1074/jbc.m207730200.

19. Lee YH, Foster J, Chen J, Voll LM, Weber APM, Tegeder M. AAP1 transports uncharged amino acids into roots of Arabidopsis. Plant J. 2007;50(2):305-19. Doi:10.1111/j.1365-313x.2007.03045.x.

20. Zhang L, Tan Q, Lee R, Trethewy A, Lee YH, Tegeder M. Altered xylem-phloem transfer of amino acids affects metabolism and leads to increased seed yield and oil content in Arabidopsis. Plant Cell. 2010;22(11):3603-20. Doi:10.1105/tpc.110.073833.

21. Okumoto S, Koch W, Tegeder M, Fischer WN, Biehl A, Leister D, Stierhof YD, Frommer WB. Root phloem-specific expression of the plasma membrane amino acid proton co-transporter AAP3. J Exp Bot. 2004;55(406):2155-68. Doi:10.1093/jxb/erh233.

22. Boorer KJ, Fischer WN. Specificity and stoichiometry of the Arabidopsis $\mathrm{H}^{+}$amino acid transporter AAP5. J Biol Chem. 1997;272(20):13040-46. Doi:10.1074/jbc.272.20.13040.

23. Schmidt R, Stransky H, Koch W. The amino acid permease AAP8 is important for early seed development in Arabidopsis thaliana. Planta. 2007;226(4):805-13. Doi:10.1007/s00425-007-0527-x.

24. Koch W, Kwart M, Laubner M, Heineke D, Stransky H, Frommer WB, Tegeder M. Reduced amino acid content in transgenic potato tubers due to antisense inhibition of the leaf $\mathrm{H}^{+}$/amino acid symporter StAAP1. Plant J. 2003;33(2):211-20. Doi:10.1046/j.1365-313X.2003.01618.x.

25. Kohl S, Hollmann J, Blattner FR, Radchuk V, Andersch F, Steuernagel B, Schmutzer T, Scholz U, Krupinska K, Weber H, et al. A putative role for amino acid permeases in sink-source communication of barley tissues uncovered by RNA-sEq. BMC Plant Biol. 2012;12(1):154. Doi:10.1186/1471-2229-12-154.

26. Ji Y, Huang W, Wu B, Fang Z, Wang X. The amino acid transporter OsAAP1 mediates growth and grain yield by regulating neutral amino acids uptake and reallocation in Oryza sativa. J Exp Bot. 2020;71(16):4763-77. Doi:10.1093/jxb/eraa256.

27. Yang Y, Chai Y, Zhang X, Lu S, Zhao Z, Wei D, Chen L, Hu YG. Multi-locus GWAS of quality traits in bread wheat: mining more candidate genes and possible regulatory network. Front Plant Sci. 2020;11:1091. Doi:10.3389/fpls.2020.01091.

28. Chen L, Bush DR. LHT1, lysine- and histidine-specific amino acid transporter in Arabidopsis. Plant Physiol. 1997;115(3):1127-34. Doi:10.1104/pp.115.3.1127.

29. Hirner A, Ladwig F, Stransky H, Okumoto S, Keinath M, Harms A, Frommer WB, Koch W. Arabidopsis LHT1 is a high-affinity transporter for cellular amino acid uptake in both root epidermis and leaf mesophyll. Plant Cell. 2006;18:1931-46. Doi:10.2307/20076748.

30. Forsum O, Svennerstam H, Ganeteg U, Näsholm T. Capacities and constraints of amino acid utilization in Arabidopsis. New Phytol. 2008;179(4):1058-69. Doi:10.1111/j.1469-8137.2008.02546.x.

31. Chen L, Ortiz-Lopez A, Jung A, Bush DR. ANT1, an aromatic and neutral amino acid transporter in Arabidopsis. Plant Physiol. 2001;125(4):1813-20. Doi:10.1104/pp.125.4.1813.

Page 12/37 
32. Marchant A, Kargul J, May ST, Muller P, Delbarre A, Perrot-Rechenmann C, Bennett MJ. AUX1 regulates root gravitropism in Arabidopsis by facilitating auxin uptake within root apical tissues. Embo J. 1999;18(8):2066-73. Doi:10.1093/emboj/18.8.2066.

33. Marchant A, Bhalerao R, Casimiro I, Eklof J, Casero PJ, Bennett M, Sandberg G. AUX1 promotes lateral root formation by facilitating indole-3-acetic acid distribution between sink and source tissues in the Arabidopsis seedling. Plant Cell. 2002;14(3):589-97. Doi:10.1105/tpc.010354.

34. Swarup R, Kargul J, Marchant A, Zadik D, Rahman A, Mills R, Yemm A, May S, Williams L, Millner P, et al. Structure function analysis of the presumptive Arabidopsis auxin permease AUX1. Plant Cell. 2004;16:3069-83. Doi:10.2307/3872286.

35. Swarup K, Benková E, Swarup R, Casimiro I, Péret B, Yang Y, Parry G, Nielsen E, Smet ID, Vanneste S, et al. The auxin influx carrier LAX3 promotes lateral root emergence. Nat Cell Biol. 2008;10(8):946-54. Doi:10.1038/ncb1754.

36. Grallath S, Weimar T, Meyer A, Gumy C, Suter-Grotemeyer M, Neuhaus JM, Rentsch D. The AtProT family. compatible solute transporters with similar substrate specificity but differential expression patterns. Plant Physiol. 2005;137(1):117-26. Doi:10.1104/pp.104.055079.

37. Lehmann S, Gumy C, Blatter E, Boeffel S, Fricke W, Rentsch D. In planta function of compatible solute transporters of the AtProT family. J Exp Bot. 2011;62(2):787-96. Doi:10.1093/jxb/erq320.

38. Mo LY, Wu LH, Tao QN. Advances in study of plant organic nitrogen nutrition. Acta Ecol Sin. 2002;22(1):118-24. (In Chinese).

39. Michaeli S, Fait A, Lagor K, Nunes-Nesi A, Grillich N, Yellin A, Bar D, Khan M, Fernie AR, Turano FJ, et al. A mitochondrial GABA permease connects the GABA shunt and the TCA cycle, and is essential for normal carbon metabolism. Plant J. 2011;67(3):485-98. Doi:10.1111/j.1365-313x.2011.04612.x.

40. Serrano R. Salt tolerance in plants and microorganisms: toxicity targets and defense responses. Int Rev Cytol. 1996;165:1-52. Doi:10.1016/S00747696(08)62219-6.

41. Lehmann S, Funck D, Szabados L, Rentsch D. Proline metabolism and transport in plant development. Amino Acids. 2010;39(4):949-62. Doi:10.1007/s00726-010-0525-3.

42. Rentsch D, Hirner B, Schmelzer E, Frommer WB. Salt stress induced proline transporters and salt stress-repressed broad specificity amino acid permeases identified by suppression of a yeast amino acid permease-targeting. Plant Cell. 1996;8(8):1437-46. Doi:10.1105/tpc.8.8.1437.

43. Guo N, Dong X, Zhang W, Zhao J, Xue C, Yan Q, Xue J, Wang H, Zhang Y, Xing H. Overexpression of GmProT1 and GmProT2 increases tolerance to drought and salt stresses in transgenic Arabidopsis. J Integr Agr. 2016;15(8):1727-43. Doi:10.1016/S2095-3119(15)61288-6.

44. Breitkreuz KE, Shelp BJ, Fischer WN, Schwacke R, Rentsch D. Identification and characterization of GABA, proline and quaternary ammonium compound transporters from Arabidopsis thaliana. FEBS Lett. 1999;450(3):280-84. Doi:10.1016/S0014-5793(99)00516-5.

45. He L, Zhang B, Wang X, Li H, Han Y. Foxtail millet: nutritional and eating quality, and prospects for genetic improvement. Front Agr Sci Eng. 2015;2(2):124-33. Doi:10.15302/j-fase-2015054.

46. Li WH, Gojobori T, Nei M. Pseudogenes as a paradigm of neutral evolution. Nature. 1981;292(5280):237-39. Doi:10.1038/292237a0.

47. Javelle M, Klein-Cosson C, Vernoud V, Boltz V, Maher C, Timmermans M, Depege-Fargeix N, Rogowsky PM. Genome-wide characterization of the HD-ZIP IV transcription factor family in maize: preferential expression in the epidermis. Plant Physiol. 2011;157(2):790-803. Doi:10.1104/pp.111.182147.

48. Hudson KA, Hudson ME. A classification of basic helix-loop-helix transcription factors of soybean. Int J Genomics. 2015;1-10. Doi:10.1155/2015/603182.

49. Tegeder M, Ward JM. Molecular evolution of plant AAP and LHT amino acid transporters. Front Plant Sci. 2012;3:21. Doi:10.3389/fpls.2012.00021.

50. Kong H, Landherr LL, Frohlich MW, Leebens-Mack J, Ma H, dePamphilis CW. Patterns of gene duplication in the plant SKP1 gene family in angiosperms: evidence for mutiple mechanisms of rapid gene birth. Plant J. 2007;50(5):873-85. Doi:10.1111/j.1365-313X.2007.03097.x.

51. Xiong LZ, Xu CG, Saghai Maroof MA, Zhang Q. Patterns of cytosine methylation in an elite rice hybrid and its parental lines, detected by a methylationsensitive amplification polymorphism technique. Mol Gen Genet. 1999;261(3):439-46. Doi:10.1007/s004380050986.

52. Dowen RH, Pelizzolaa M, Schmitza RJ, Listera R, Dowen JM, Nerya JR, Dixon JE, Ecker JR. Widespread dynamic DNA methylation in response to biotic stress. P Natl Acad Sci USA. 2012;109(32):2183-91. Doi:10.1073/pnas.1209329109.

53. Takuno S, Gaut BS. Gene body methylation is conserved between plant orthologs and is of evolutionary consequence. P Natl Acad Sci USA. 2013;110(5):1791-802. Doi:10.1073/pnas.1215380110.

54. Grewal SIS, Moazed D. Heterochromatin and epigenetic control of gene expression. Science. 2003;301(5634):798-802. Doi:10.1126/science.1086887.

55. Ludwig M. Evolution of the C4 photosynthetic pathway: events at the cellular and molecular levels. Photosynth Res. 2013;117(1-3):147-61. Doi:10.1007/s11120-013-9853-y.

56. Zhang G, Liu X, Quan Z, Cheng S, Xu X, Pan S, Xie M, Zeng P, Yue Z, Wang W, et al. Genome sequence of foxtail millet (Setaria italica) provides insights into grass evolution and biofuel potential. Nat Biotechnol. 2012;30(6):549-54. Doi:10.1038/nbt.2195.

57. Schilling S, Kennedy A, Pan S, Jermiin LS, Melzer R. Genome-wide analysis of MIKC-type MADS-box genes in wheat: pervasive duplications, functional conservation and putative neofunctionalization. New Phytol. 2019;225(1):511-29. Doi:10.1111/nph.16122.

58. Hirner B, Fischer WN, Rentsch D, Kwart M, Frommer WB. Developmental control of H+/amino acid permease gene expression during seed development of Arabidopsis. Plant J. 1998;14(5):535-44. Doi:10.1046/j.1365-313X.1998.00151.x.

59. Popova OV, Dietz KJ, Golldack D. Salt-dependent expression of a nitrate transporter and two amino acid transporter genes in Mesembryanthemum crytallinum. Plant Mol Biol. 2003;52(3):569-78. Doi:10.1023/A:1024802101057.

60. Ueda A, Shi W, Sanmiya K, Shono M, Takabe T. Functional analysis of salt-induced proline transporter of barely roots. Plant cell Physiol. 2001;42(11):1282-89. Doi:10.1093/pcp/pce166.

Page $13 / 37$ 
61. Schmidt R, Stransky H, Koch W. The amino acid permease AAP8 is important for early seed development in Arabidopsis thaliana. Planta. 2007;226(4):805-13. Doi:10.1007/s00425-007-0527-x.

62. Rolletschek H, Hosein F, Miranda M, Heim U, Gotz KP, Schlereth A, Borisjuk L, Saalbach I, Wobus U, Weber H. Ectopic expression of an amino acid transporter (VfAAP1) in seeds of Vicia narbonensis and pea increases storage proteins. Plant Physiol. 2005;137(4):1236-49. Doi:10.1104/pp.104.056523.

63. Svennerstam H, Ganeteg U, Bellini C, Nasholm T. Comprehensive screening of Arabidopsis mutants suggests the lysine histidine transporter 1 to be involved in plant uptake of amino acids. Plant Physiol. 2007;143(4):1853-60. Doi:10.1104/pp.106.092205.

64. Wheeler TJ, Eddy SR. nhmmer: DNA homology search with profile HMMs. Bioinformatics. 2013;29(19):2487-89. Doi:10.1093/bioinformatics/btt403.

65. Gasteiger E, Hoogland C, Gattiker A, Duvaud Se, Wilkins MR, Appel RD, Bairoch A. Protein identification and analysis tools on the ExPASy server. The Proteomics Protocols Handbook. 2005;571-607. Doi:10.1385/1-59259-890-0:571.

66. Goldberg T, Hecht M, Hamp T, Karl T, Yachdav G, Ahmed N, Altermann U, Angerer P, Ansorge S, Balasz K, et al. LocTree3 prediction of localization. Nucleic Acids Res. 2014;42(W1):W350-5. Doi:10.1093/nar/gku396.

67. Wang Y, Tang H, Debarry JD, Tan X, Li J, Wang X, Lee TH, Jin H, Marler B, Guo H, et al. MCScanX: a toolkit for detection and evolutionary analysis of gene synteny and collinearity. Nucleic Acids Res. 2012;40(7):e49. Doi:10.1093/nar/gkr1293.

68. Wang M, Yue H, Feng K, Deng P, Song W, Nie X. Genome-wide identification, phylogeny and expressional profiles of mitogen activated protein kinase kinase kinase (MAPKKK) gene family in bread wheat (Triticum aestivum L.). BMC Genom. 2016;17(1):668. Doi:10.1186/s12864-016-2993-7.

69. Chen C, Xia R, Chen H, He Y. TBtools, a Toolkit for Biologists integrating various HTS-data handling tools with a user-friendly interface. bioRxiv. 2018;289660. Doi:10.1101/289660.

70. Wang D, Zhang Y, Zhang Z, Zhu J, Yu J. KaKs_Calculator 2.0: a toolkit incorporating gamma-series methods and sliding window strategies. Genom Proteom Bioinf. 2010;8(1):77-80. Doi:10.1016/S1672-0229(10)60008-3.

71. Tamura K, Stecher G, Peterson D, Filipski A, Kumar S. MEGA6: Molecular Evolutionary Genetics Analysis Version 6.0. Mol Biol Evol. 2013;30(12):2725-29. Doi:10.1093/molbev/mst197.

72. Bailey TL, Boden M, Buske FA, Frith M, Grant CE, Clementi L, Ren J, Li WW, Noble WS. MEME SUITE: tools for motif discovery and searching. Nucleic Acids Res. 2009;37(Web Server):W202-8. Doi:10.1093/nar/gkp335.

73. Robert X, Gouet P. Deciphering key features in protein structures with the new ENDscript server. Nucleic Acids Res. 2014;42(W1):W320-4. Doi:10.1093/nar/gku316.

74. Emms DM, Kelly S. OrthoFinder: solving fundamental biases in whole genome comparisons dramatically improves orthogroup inference accuracy. Genome Biol. 2015;16(1):157. Doi:10.1186/s13059-015-0721-2.

75. Robinson MD, McCarthy DJ, Smyth GK. edgeR: a Bioconductor package for differential expression analysis of digital gene expression data. Bioinformatics. 2010;26(1):139-40. Doi:10.1093/bioinformatics/btp616.

76. Nicolaides NC, Stoeckert CJA, Simple. Efficient Method for the Separate Isolation of RNA and DNA from the Same Cells. Bio Techniques. 1990;8(2):154-6.

77. Pertea M, Kim D, Pertea GM, Leek JT, Salzberg SL. Transcript-level expression analysis of RNA-seq experiments with HISAT, StringTie and Ballgown. Nat Protoc. 2016;11(9):1650-67. Doi:10.1038/nprot.2016.095.

78. Wang L, Feng Z, Wang X, Wang X, Zhang X. DEGseq: an R package for identifying differentially expressed genes from RNA-seq data. Bioinformatics. 2009;26(1):136-8. Doi:10.1093/bioinformatics/btp612.

79. Trapnell C, Williams BA, Pertea G, Mortazavi A, Kwan G, van Baren MJ, Salzberg SL, Wold BJ, Pachter L. Transcript assembly and quantification by RNASeq reveals unannotated transcripts and isoform switching during cell differentiation. Nat Biotechnol. 2010;28(5):511-5. Doi:10.1038/nbt.1621.

80. Kanehisa M, Araki M, Goto S, Hattori M, Hirakawa M, Itoh M, Katayama T, Kawashima S, Okuda S, Tokimatsu T, et al. KEGG for linking genomes to life and the environment. Nucleic Acids Res. 2008;36(Database):D480-4. Doi:10.1093/nar/gkm882.

81. Qi X, Xie S, Liu Y, Yi F, Yu J. Genome-wide annotation of genes and noncoding RNAs of foxtail millet in response to simulated drought stress by deep sequencing. Plant Mol Biol. 2013;83(4-5):459-73. Doi:10.1007/s11103-013-0104-6.

82. Pan J, Li Z, Dai S, Ding H, Wang Q, Li X, Ding G, Wang P, Guan Y, Liu W. Integrative analyses of transcriptomics and metabolomics upon seed germination of foxtail millet in response to salinity. Sci Rep. 2020;10(1):13660. Doi:10.1038/s41598-020-70520-1.

\section{Figures}


a

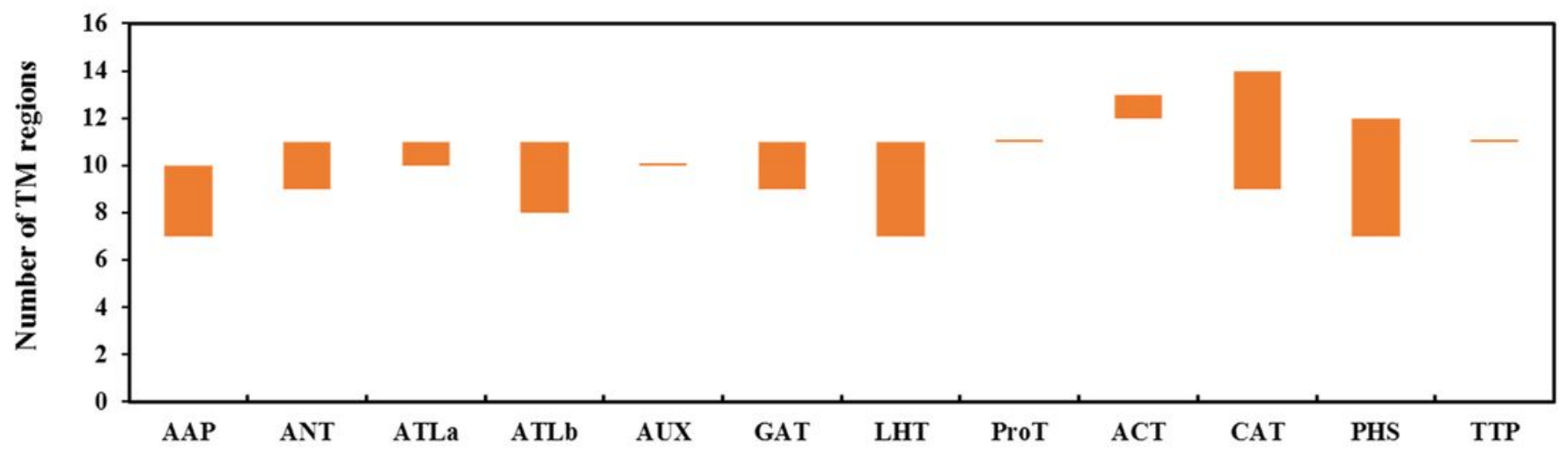

b

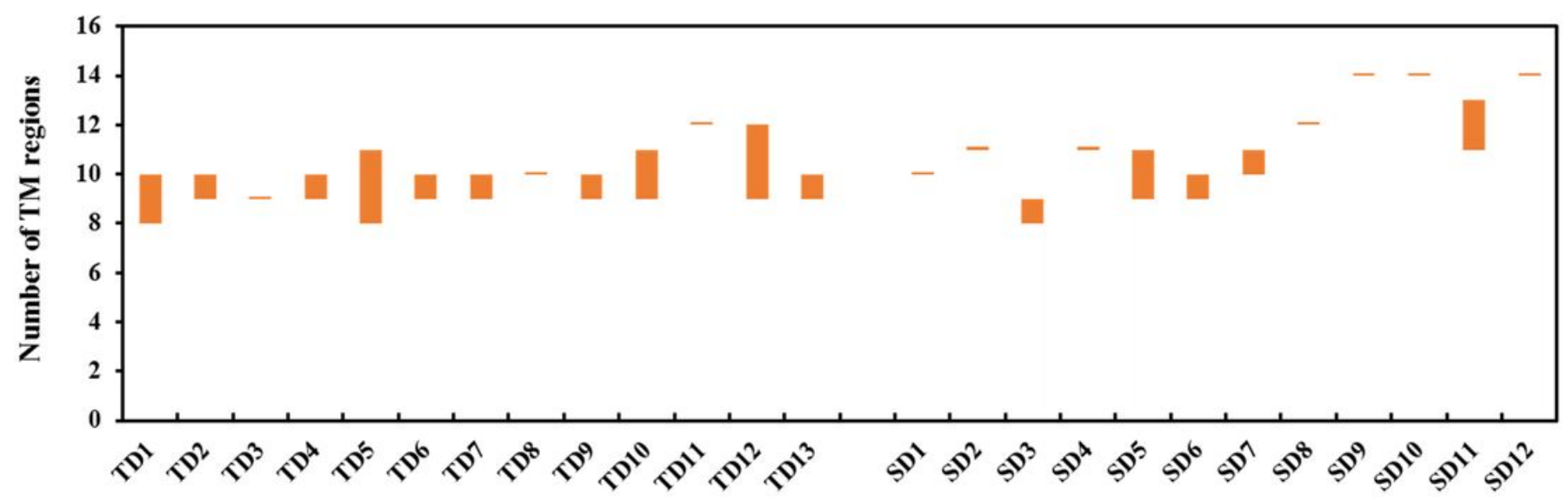

Figure 1

The variations in the number of putative transmembrane (TM) regions of AAT proteins in foxtail millet. (a) The X-axis and Y-axis represent the 12 AAT subfamily members and the number of TM regions, respectively; (b) The X-axis lists all duplicated gene groups, and $\mathrm{Y}$-axis represents the number of TM regions. 
a

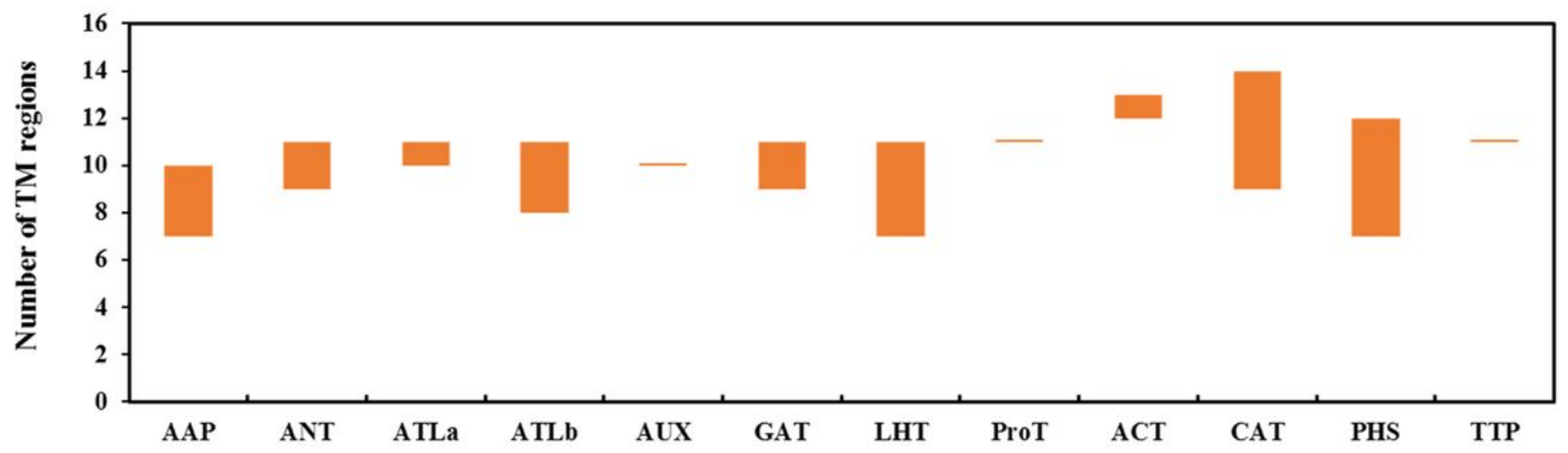

b

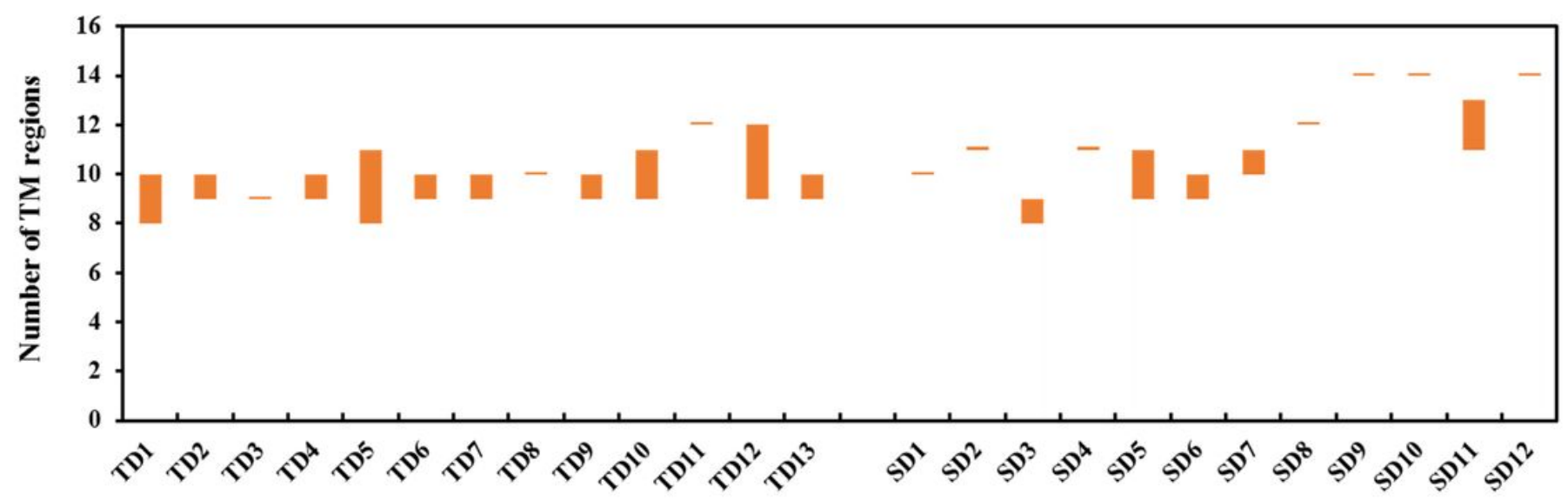

Figure 1

The variations in the number of putative transmembrane (TM) regions of AAT proteins in foxtail millet. (a) The X-axis and Y-axis represent the 12 AAT subfamily members and the number of TM regions, respectively; (b) The X-axis lists all duplicated gene groups, and $\mathrm{Y}$-axis represents the number of TM regions. 


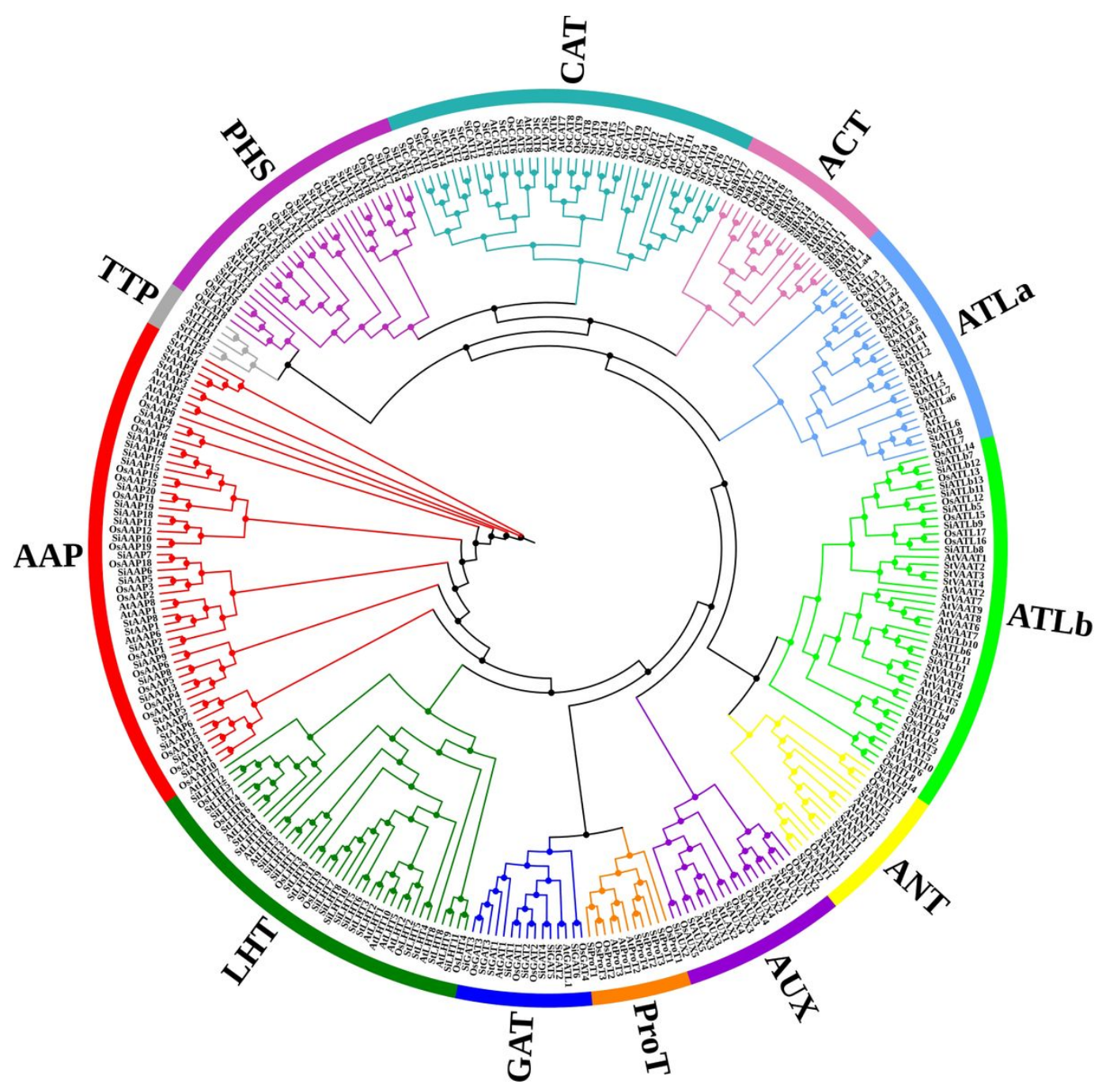

Figure 2

Phylogenetic tree constructed by the AAT proteins of foxtail millet, rice, Arabidopsis and potato. MUSCLE and MEGA 6.06 were used to perform the multiple sequence alignment and construct the phylogenetic tree with the maximum likelihood (ML) method and the JTT model. Different AAT subfamily members were shown with different colored branches. AAT proteins were named according to the ID in published articles, such as OsAAP1, AtAAP1 and StAAP1. 


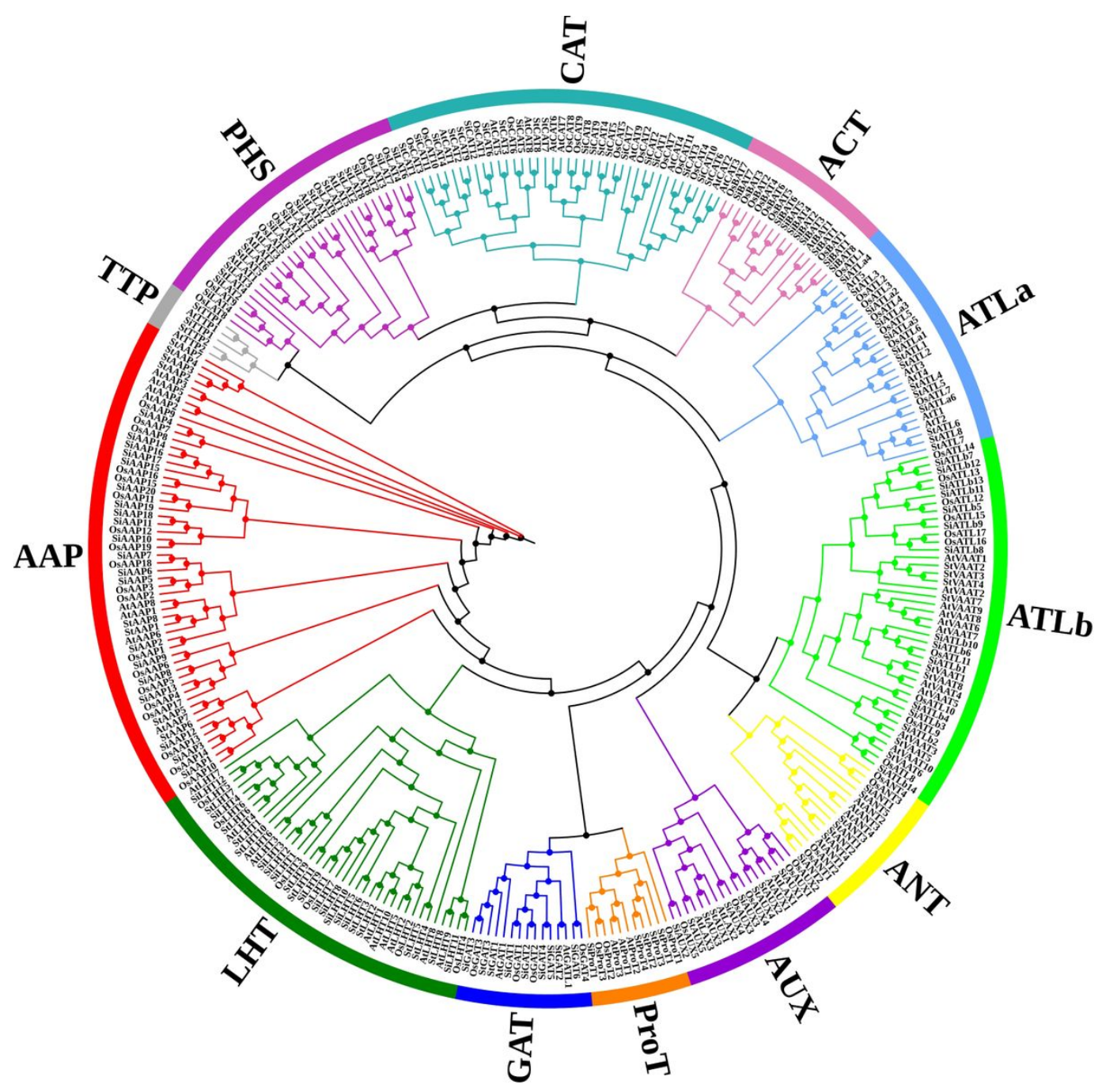

Figure 2

Phylogenetic tree constructed by the AAT proteins of foxtail millet, rice, Arabidopsis and potato. MUSCLE and MEGA 6.06 were used to perform the multiple sequence alignment and construct the phylogenetic tree with the maximum likelihood (ML) method and the JTT model. Different AAT subfamily members were shown with different colored branches. AAT proteins were named according to the ID in published articles, such as OsAAP1, AtAAP1 and StAAP1. 

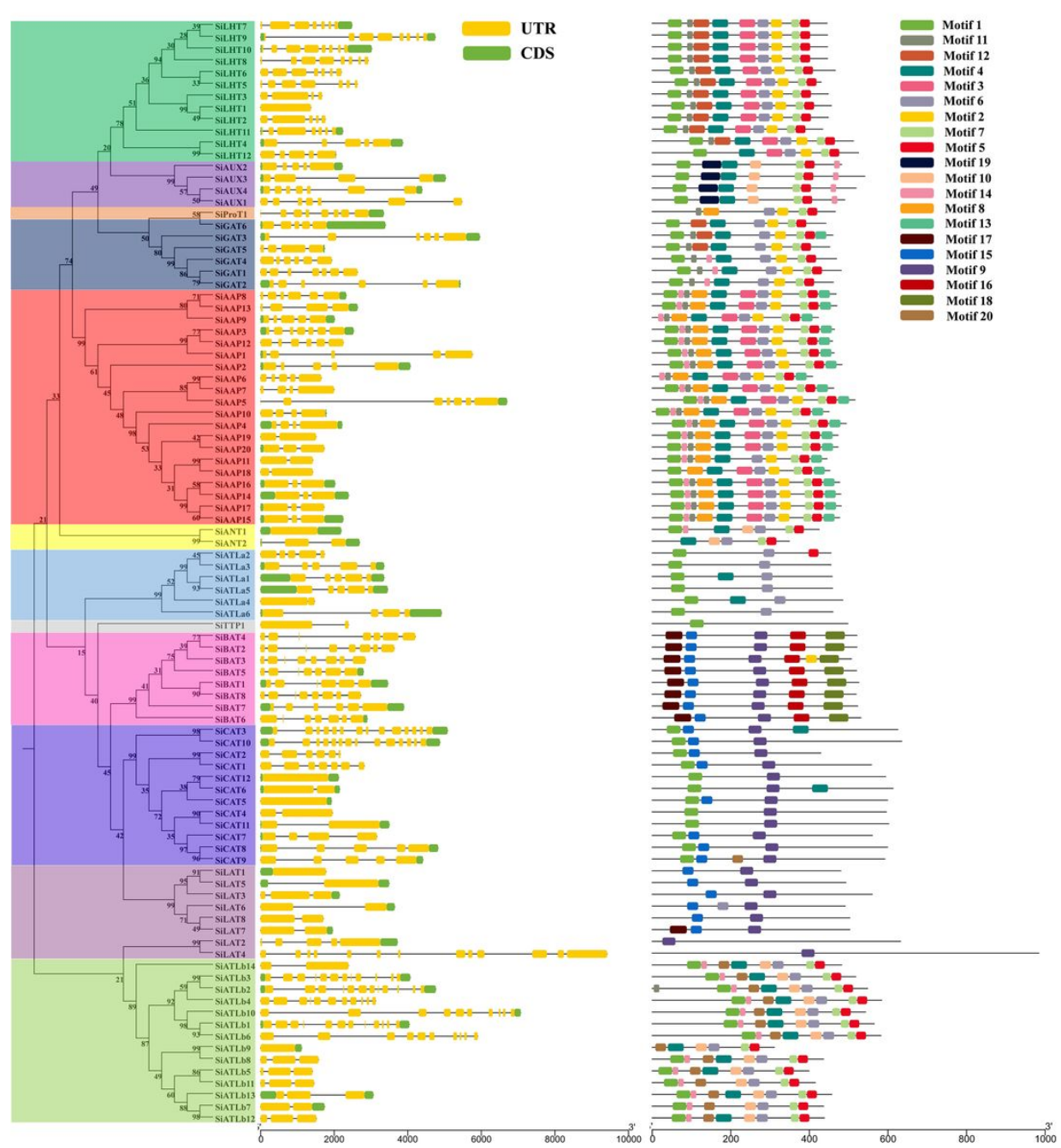

\section{Figure 3}

Gene structure and conserved motifs of SiAATs in each subfamily. Different subfamilies of foxtail millet AAT family are shown with various color backgrounds. The gene structure was referred from the gene model file of foxtail millet reference genome. The exons, UTR regions and introns are displayed by green boxes, yellow boxes and the block lines, respectively. The detail motif sequences are as listed in Additional file 2: Figure S2. 

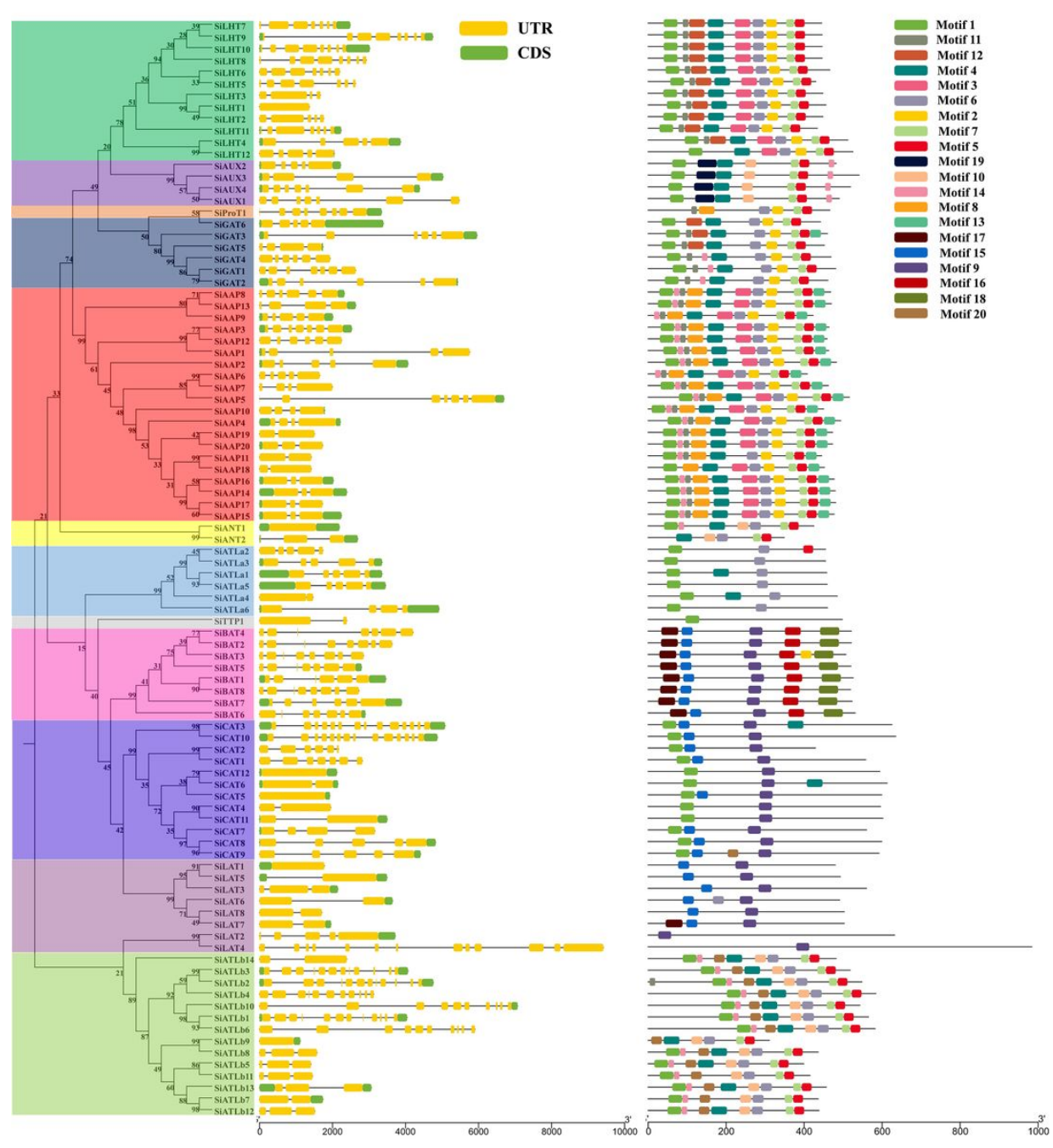

\section{Figure 3}

Gene structure and conserved motifs of SiAATs in each subfamily. Different subfamilies of foxtail millet AAT family are shown with various color backgrounds. The gene structure was referred from the gene model file of foxtail millet reference genome. The exons, UTR regions and introns are displayed by green boxes, yellow boxes and the block lines, respectively. The detail motif sequences are as listed in Additional file 2: Figure S2. 

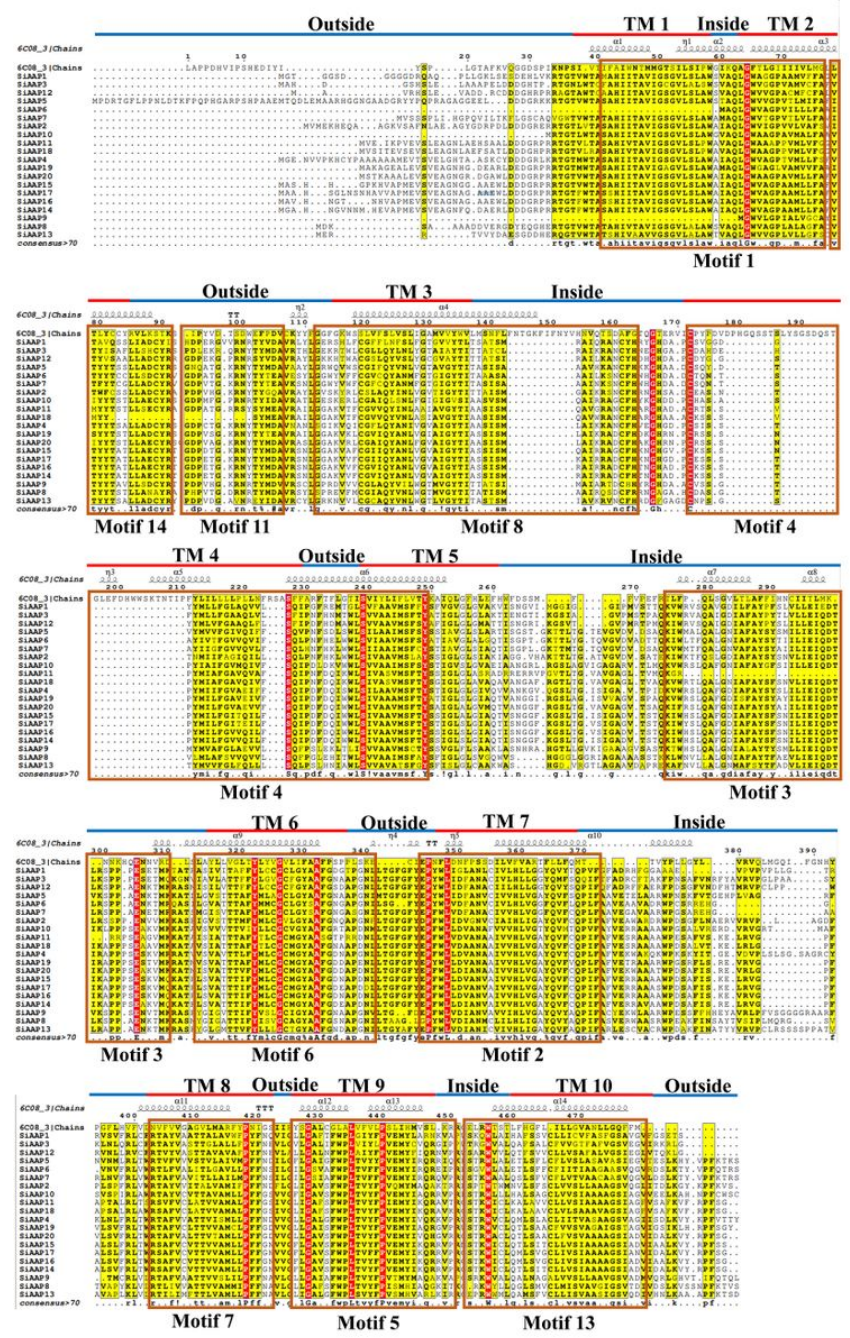

\section{Figure 4}

The multiple-sequence alignment of the AAP proteins in foxtail millet. The TM regions of SiAAP proteins are indicated with red line, and their conserved motifs predicted by MEME are shown in the red boxes. 

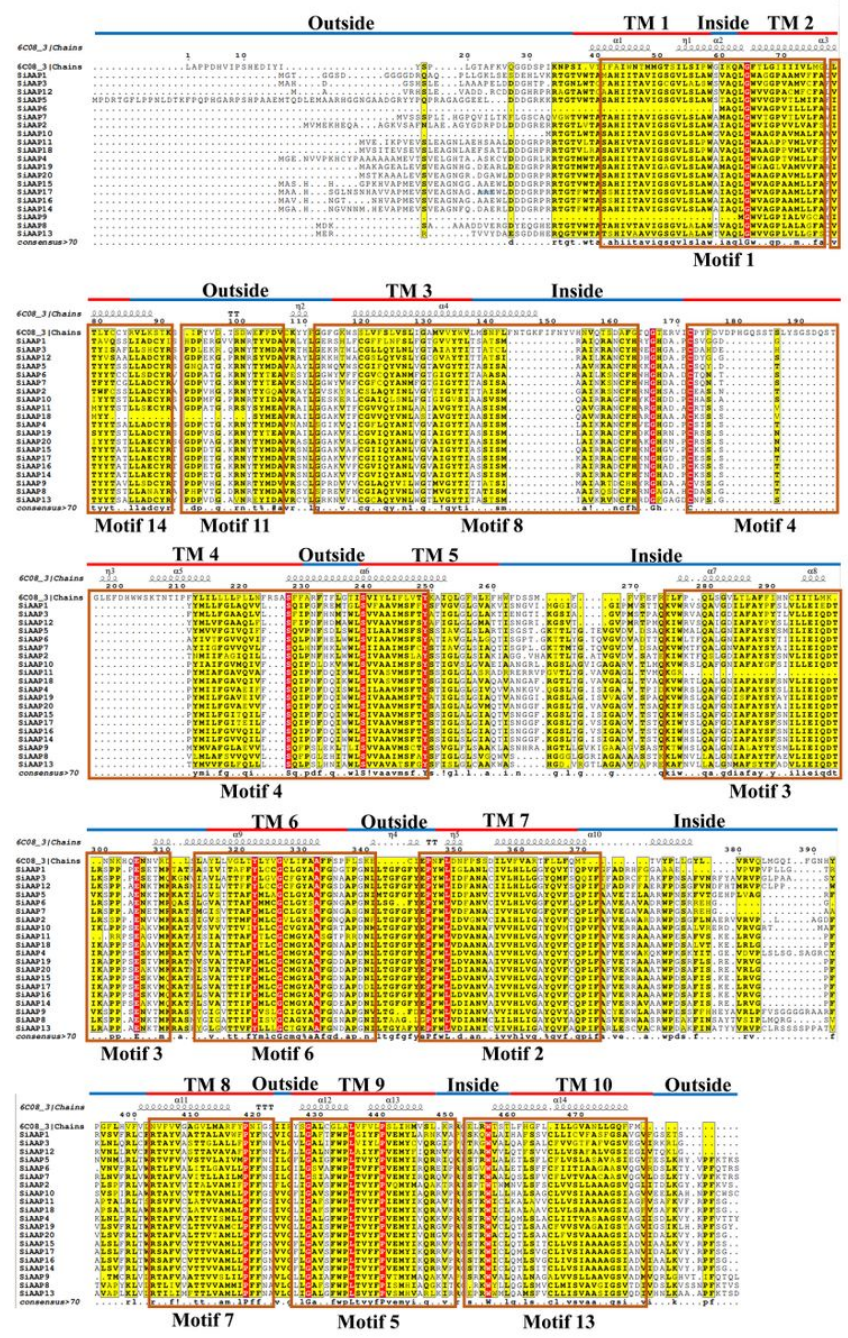

\section{Figure 4}

The multiple-sequence alignment of the AAP proteins in foxtail millet. The TM regions of SiAAP proteins are indicated with red line, and their conserved motifs predicted by MEME are shown in the red boxes. 
0.75

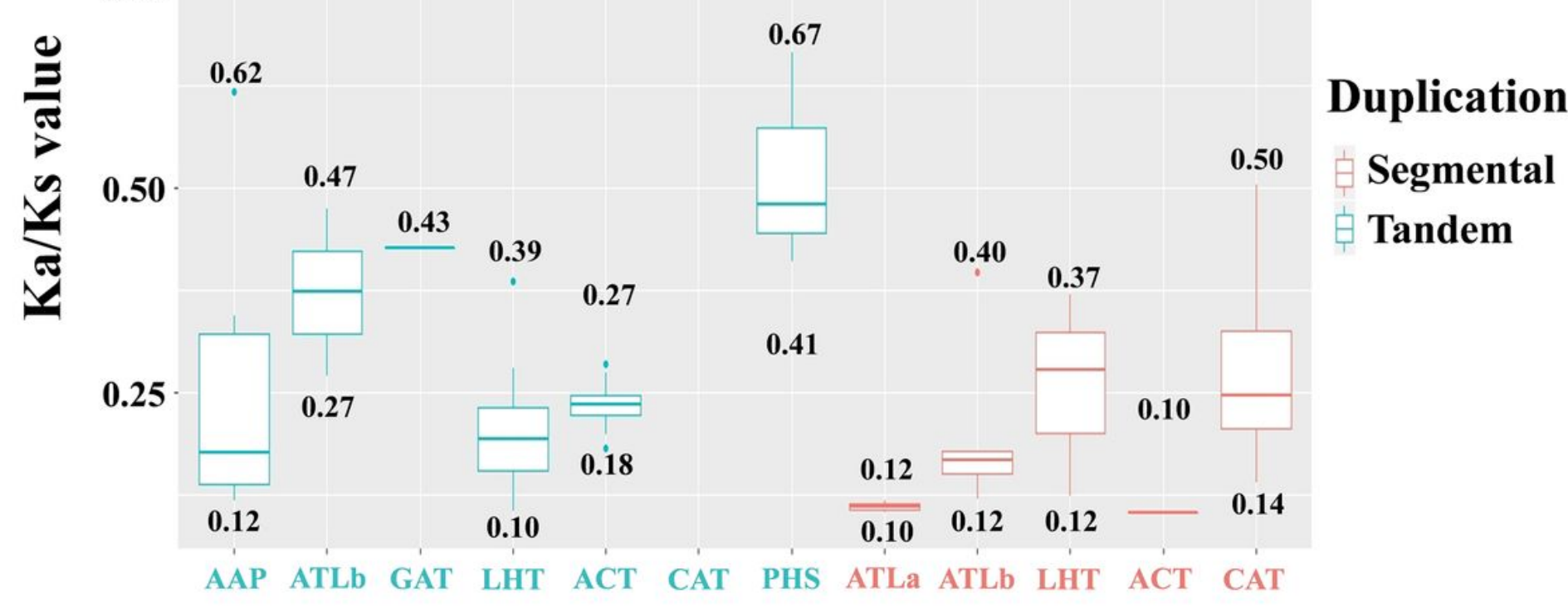

Figure 5

The Ka/Ks values of homologous AAT gene groups in different AAT subfamilies of foxtail millet. The X-axis and Y-axis indicates the duplicated AAT gene pairs in different subfamilies and Ka/Ks (nonsynonymous / synonymous) values, respectively. Segmental duplications and tandem duplications were shown in red and blue, respectively.

1.00

0.97

0.75

$\frac{0}{3}$

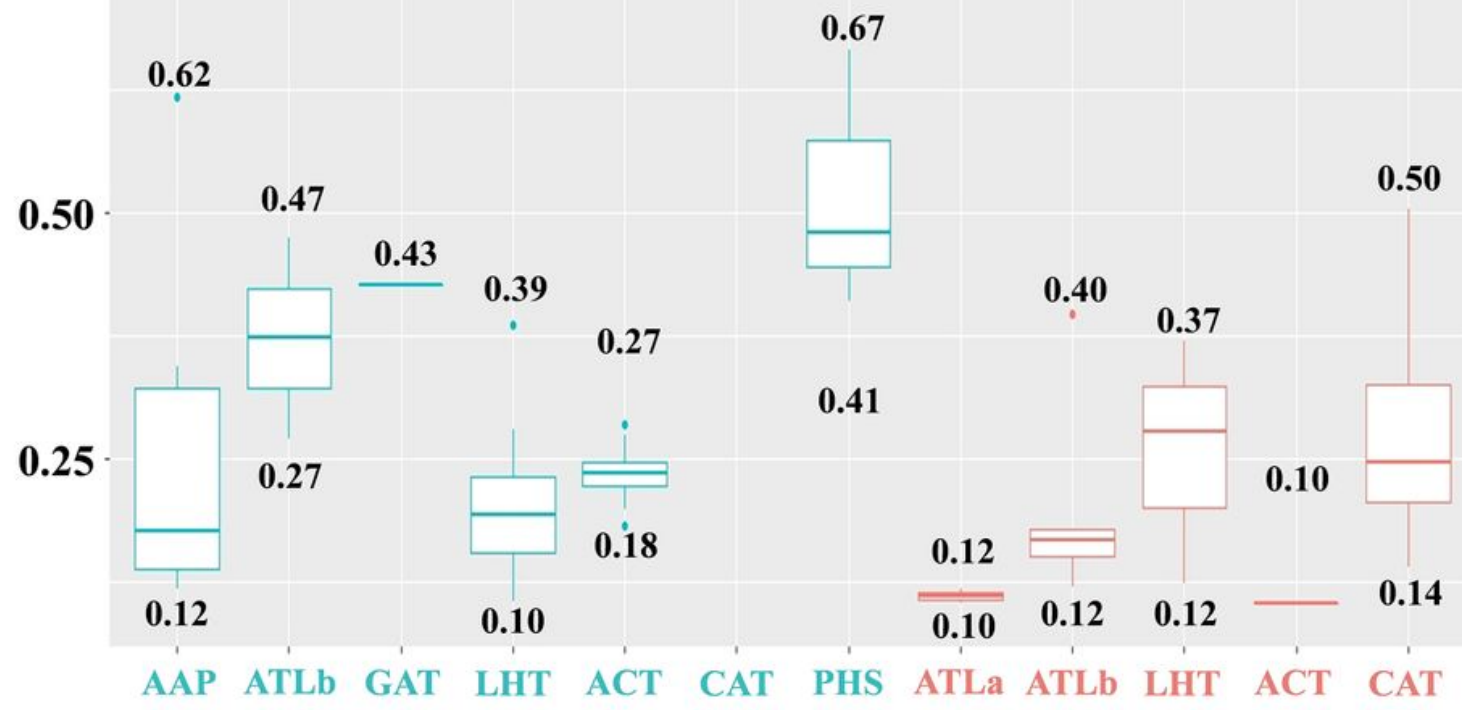

Duplication 
The Ka/Ks values of homologous AAT gene groups in different AAT subfamilies of foxtail millet. The X-axis and Y-axis indicates the duplicated AAT gene pairs in different subfamilies and Ka/Ks (nonsynonymous / synonymous) values, respectively. Segmental duplications and tandem duplications were shown in red and blue, respectively.

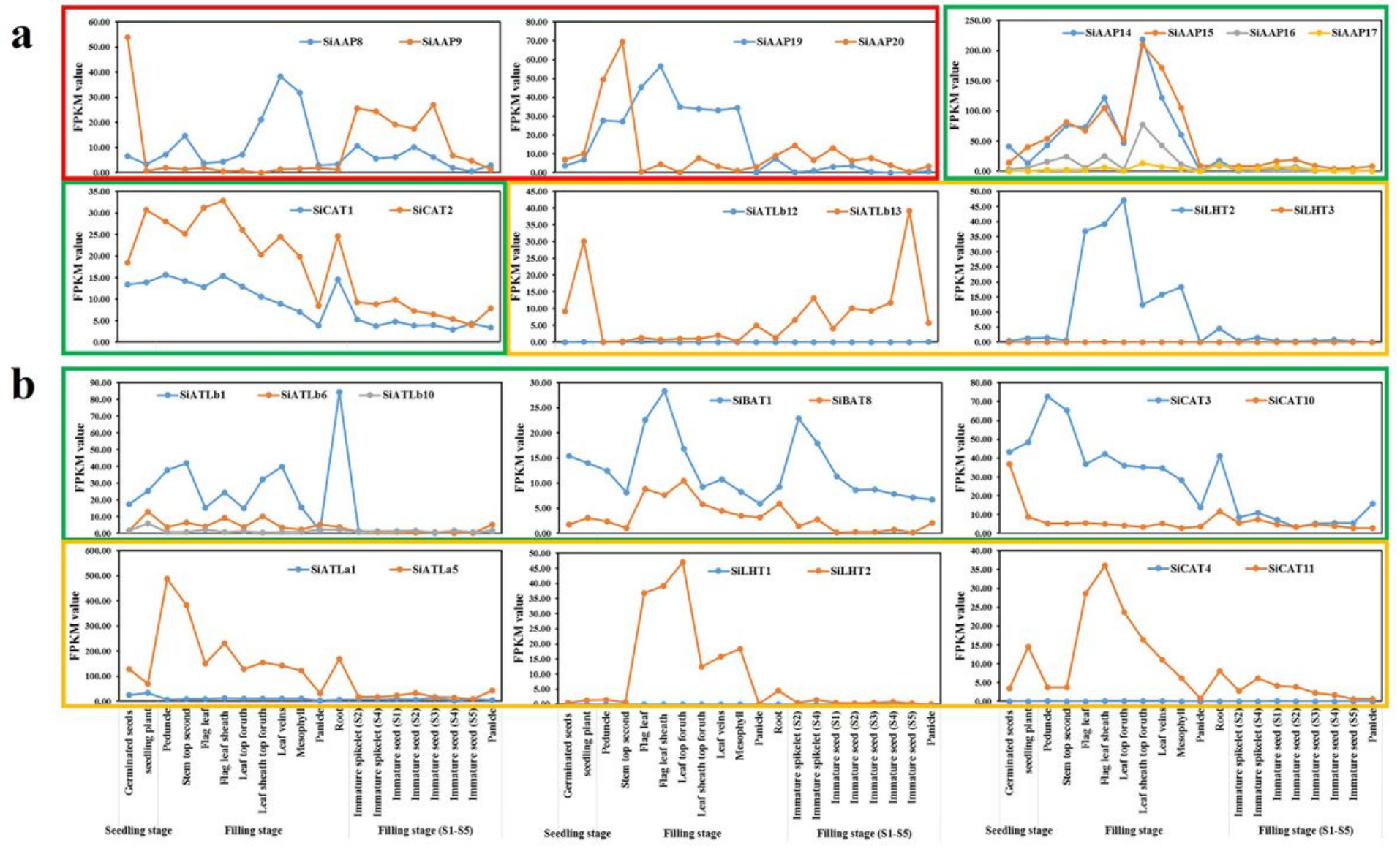

Figure 6

Three variations of expression patterns of duplicated AAT gene groups in foxtail millet. (a) and (b) is for the tandemly and segmentally duplicated gene groups, respectively. The X-axis indicates different tissue at different stage, and the Y-axis represents the FPKM value. Different colored boxes represent different expression patterns. Red boxes: new-functionalization; Blue boxes: sub-functionalization; Yellow boxes: non-functionalization. 
a
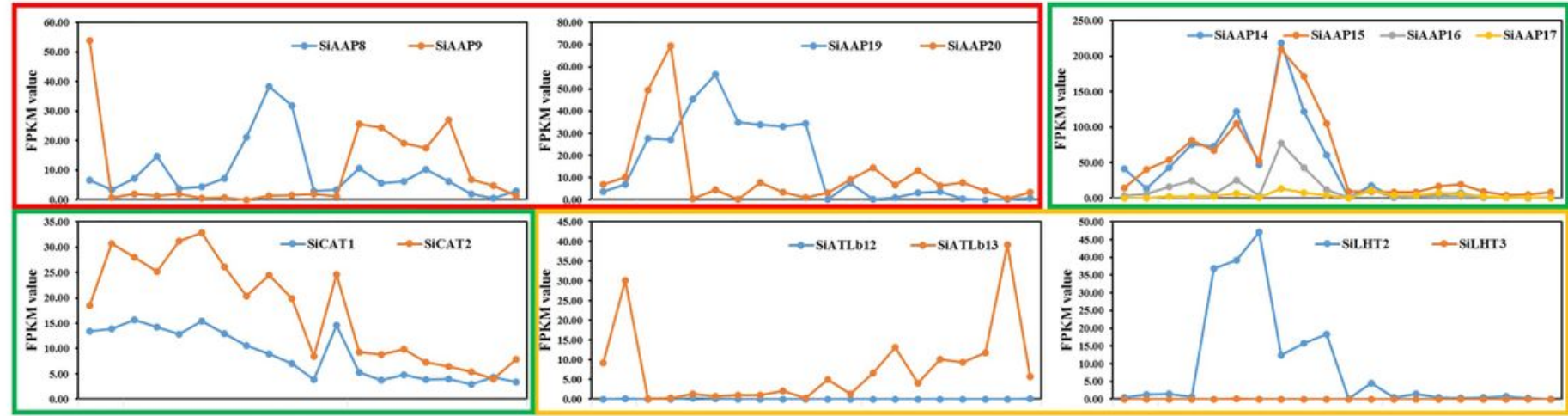

b
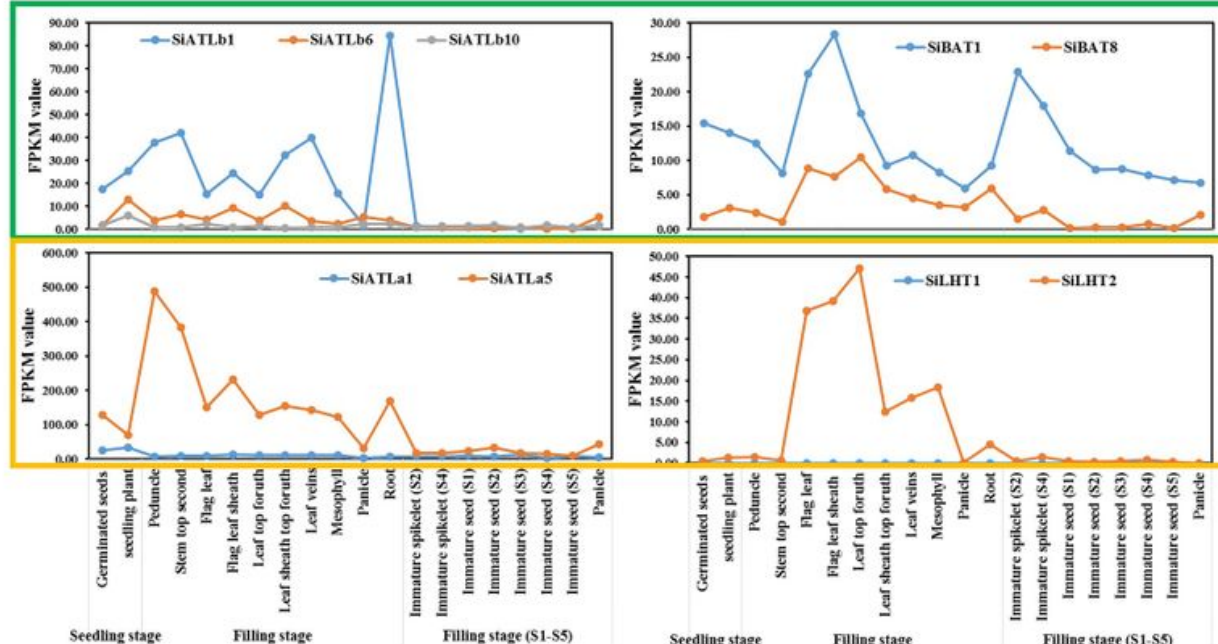

Seedling stage
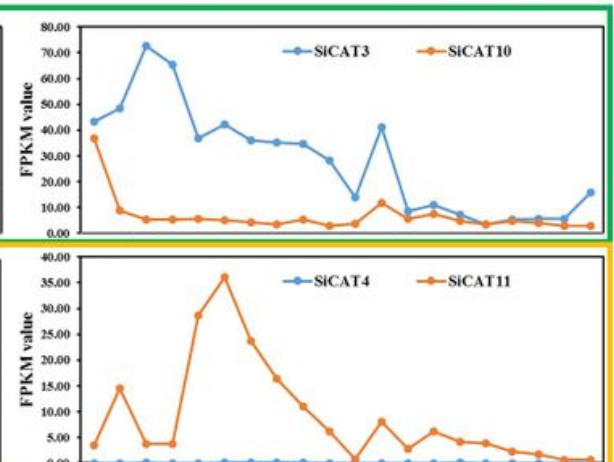

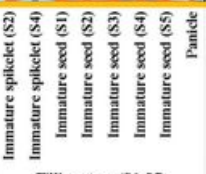

Filling stage (S1-ss)

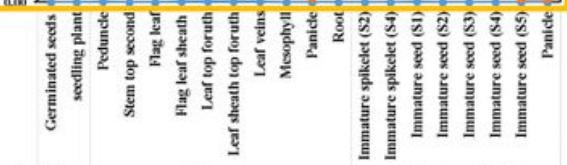

Seedling stage Filling stage

Figure 6

Three variations of expression patterns of duplicated AAT gene groups in foxtail millet. (a) and (b) is for the tandemly and segmentally duplicated gene groups, respectively. The $\mathrm{X}$-axis indicates different tissue at different stage, and the $\mathrm{Y}$-axis represents the FPKM value. Different colored boxes represent different expression patterns. Red boxes: new-functionalization; Blue boxes: sub-functionalization; Yellow boxes: non-functionalization. 

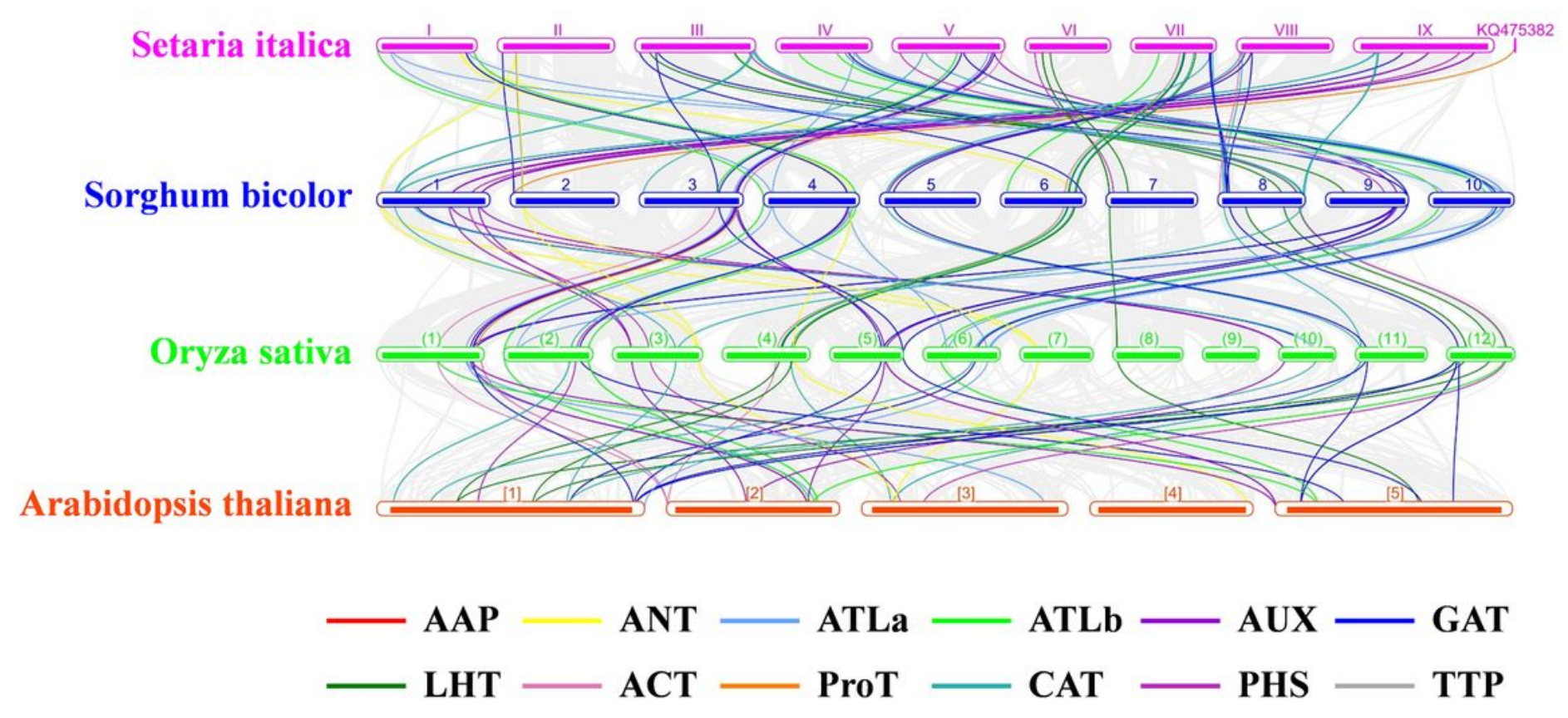

Figure 7

Collinearity of the orthologous AAT genes in foxtail millet, sorghum, rice and Arabidopsis. The genome of each species is shown in one row, and the AAT genes of different subfamily are shown with different colored lines. The collinear relationship of all orthologous genes in different species was shown with the gray lines. 

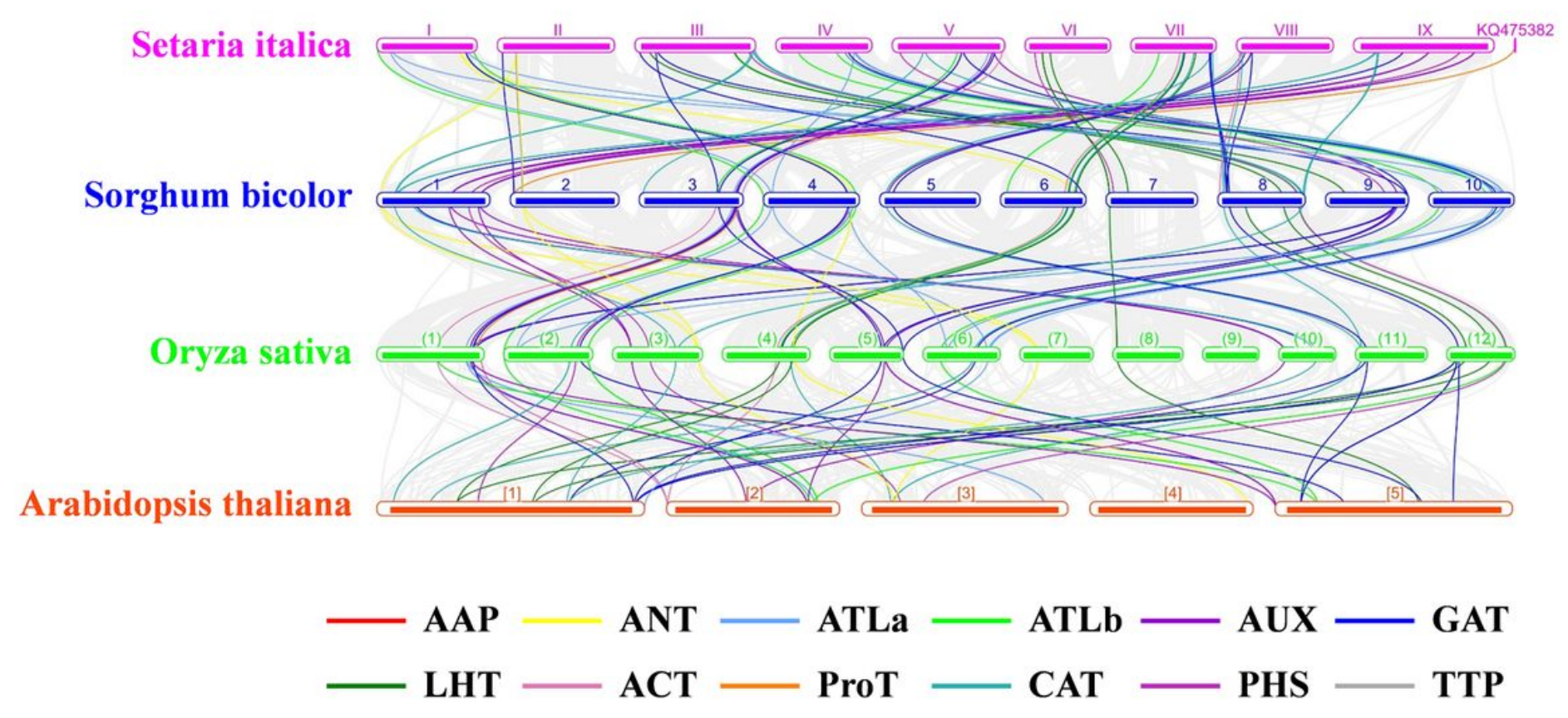

Figure 7

Collinearity of the orthologous AAT genes in foxtail millet, sorghum, rice and Arabidopsis. The genome of each species is shown in one row, and the AAT genes of different subfamily are shown with different colored lines. The collinear relationship of all orthologous genes in different species was shown with the gray lines. 

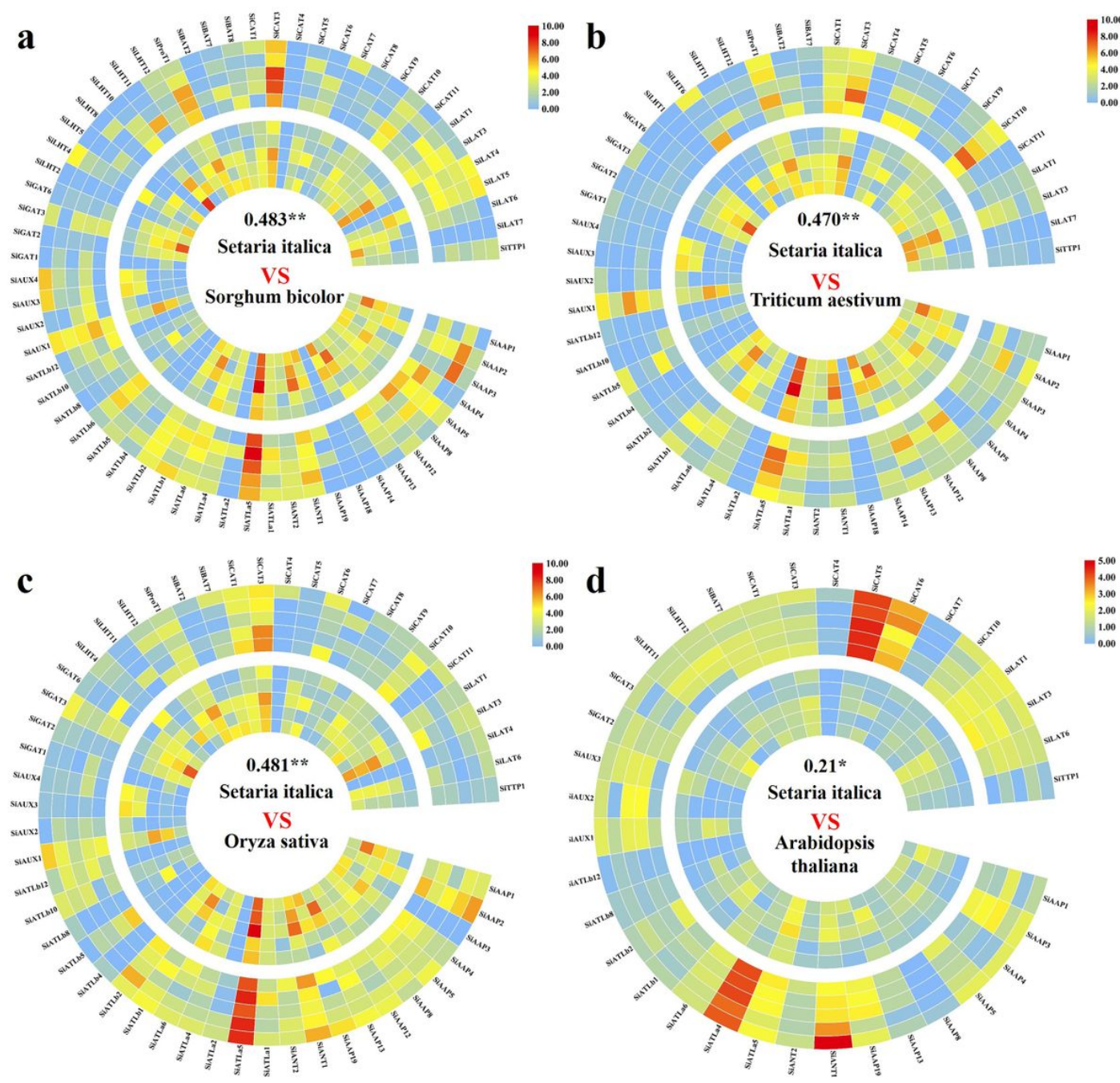

\section{Figure 8}

Comparison on the expressions of orthologous AAT genes in five main tissues between foxtail millet with other 4 species. The outside and the inside rings of (a) to (d) represent foxtail millet and another species, respectively. From the outside to the inside of each ring are the expression levels of AAT genes in leaf, root, stem, grain and spike/inflorescence, respectively. The numbers in the circles represent the correlation coefficients between the expressions of orthologous AAT genes in the two species. * and ** represent correlation significant at $\mathrm{P}<0.05$ and $\mathrm{P}<0.01$ probability level, respectively. 

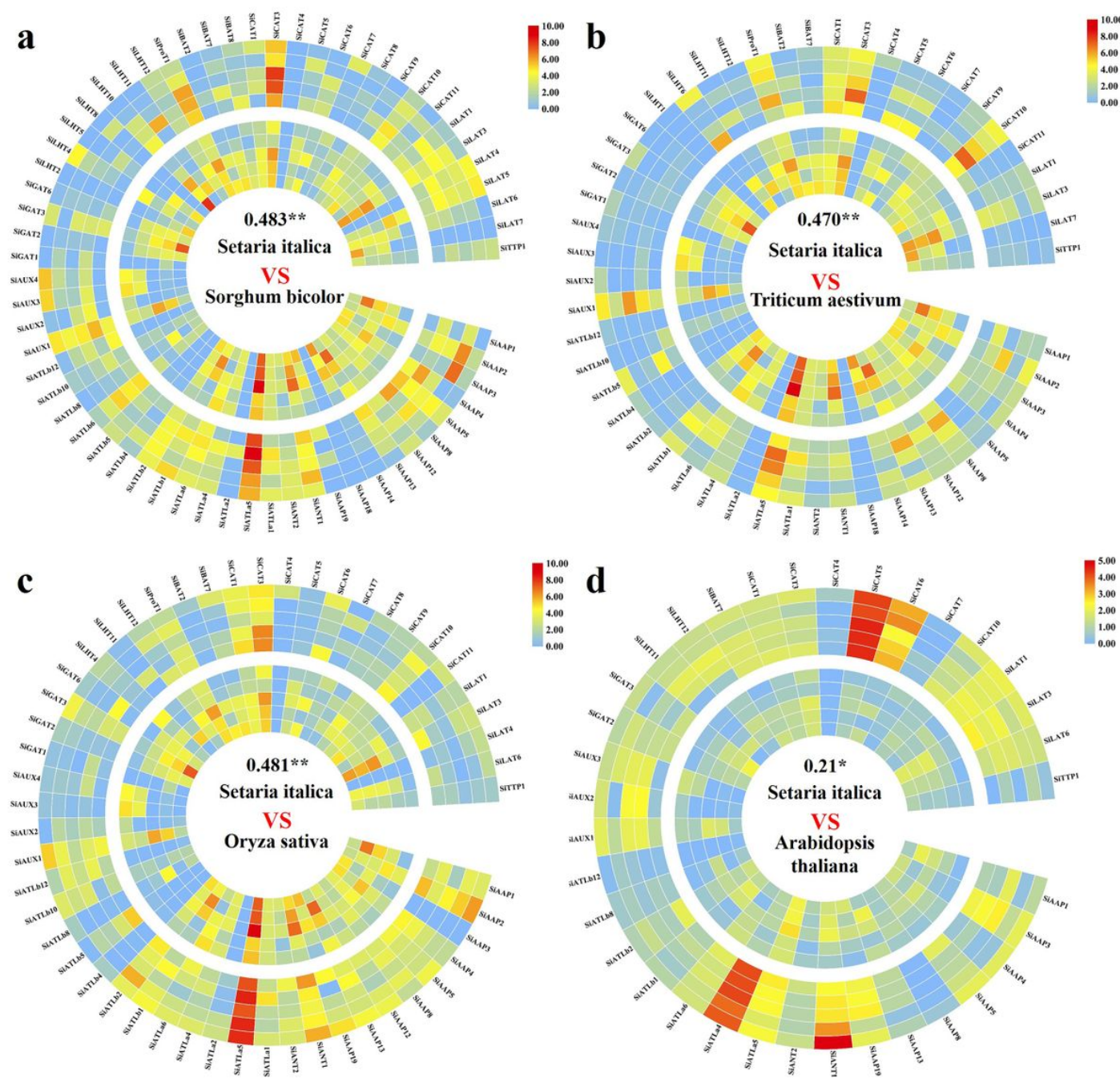

\section{Figure 8}

Comparison on the expressions of orthologous AAT genes in five main tissues between foxtail millet with other 4 species. The outside and the inside rings of (a) to (d) represent foxtail millet and another species, respectively. From the outside to the inside of each ring are the expression levels of AAT genes in leaf, root, stem, grain and spike/inflorescence, respectively. The numbers in the circles represent the correlation coefficients between the expressions of orthologous AAT genes in the two species. * and ** represent correlation significant at $\mathrm{P}<0.05$ and $\mathrm{P}<0.01$ probability level, respectively. 


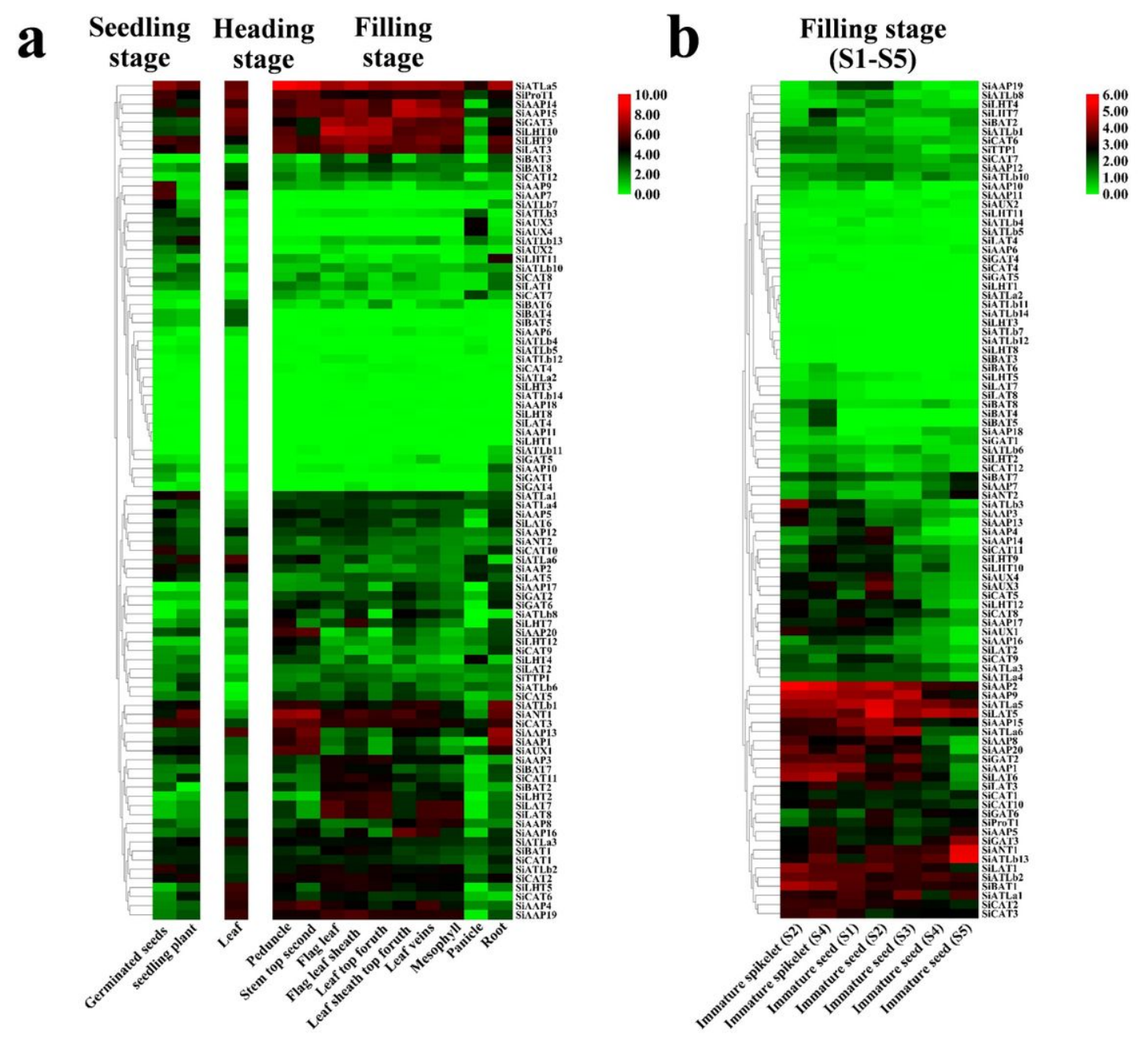

Figure 9

Expression patterns of SiAATs in multiple tissues and developing grain of foxtail millet. (a) The expression patterns of SiAAT genes in 13 tissues at three growth stages, which were reproduced based on the transcriptome data; (b) The expression pattern of SiAATs in developing grain, which were obtained from RNA-seq. Tissue name is listed at the bottom of each column, while the color scale is shown at the right. The log2 (FPKM+1) values are used to display the expression levels. 

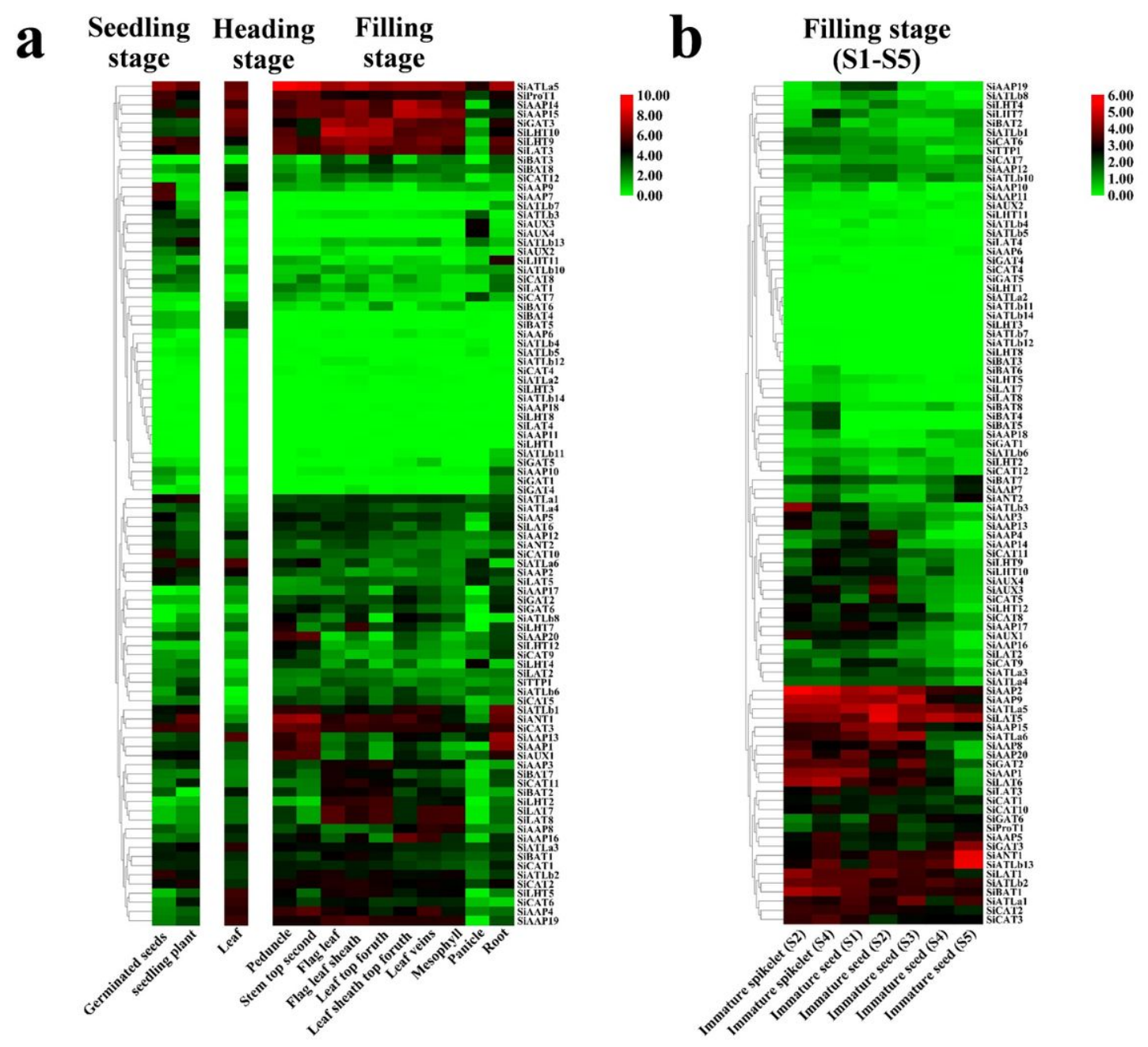

Figure 9

Expression patterns of SiAATs in multiple tissues and developing grain of foxtail millet. (a) The expression patterns of SiAAT genes in 13 tissues at three growth stages, which were reproduced based on the transcriptome data; (b) The expression pattern of SiAATs in developing grain, which were obtained from RNA-seq. Tissue name is listed at the bottom of each column, while the color scale is shown at the right. The log2 (FPKM+1) values are used to display the expression levels.

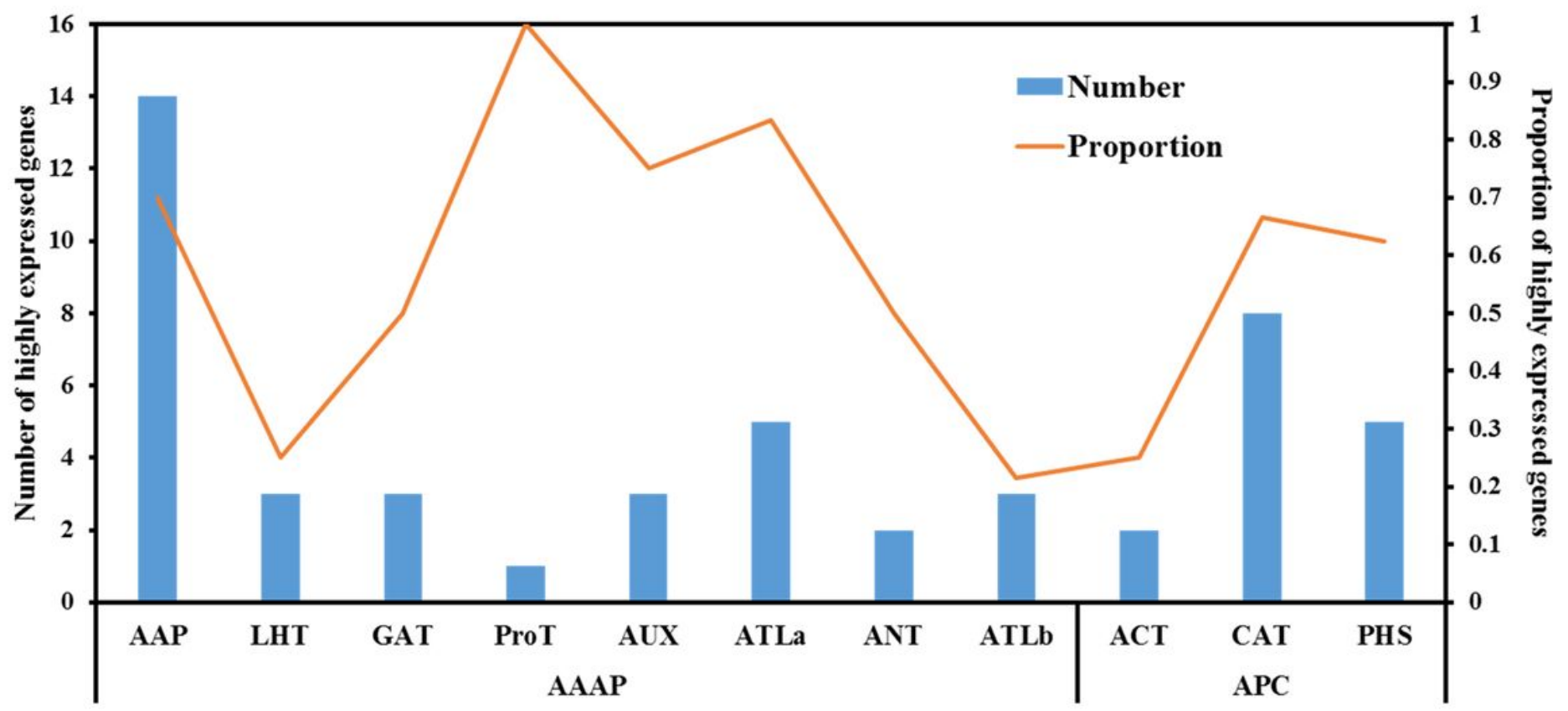


The numbers and proportions of the SiAAT genes in different subfamilies specifically expressed in grain development. The bars represent the number of genes in different subfamilies, and the broken line represents the rate of grain-specific expressed genes to the total number of genes in the subfamily.

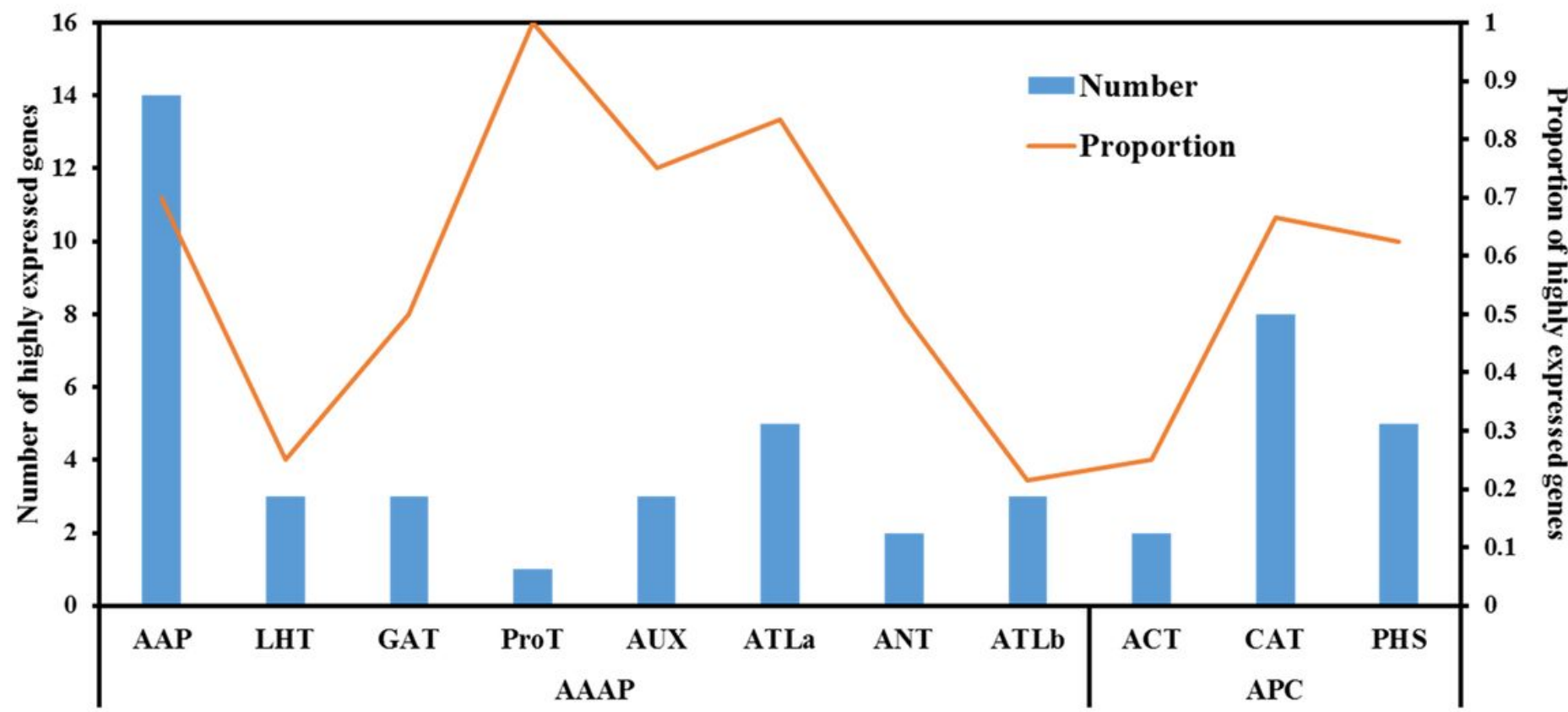

Figure 10

The numbers and proportions of the SiAAT genes in different subfamilies specifically expressed in grain development. The bars represent the number of genes in different subfamilies, and the broken line represents the rate of grain-specific expressed genes to the total number of genes in the subfamily.

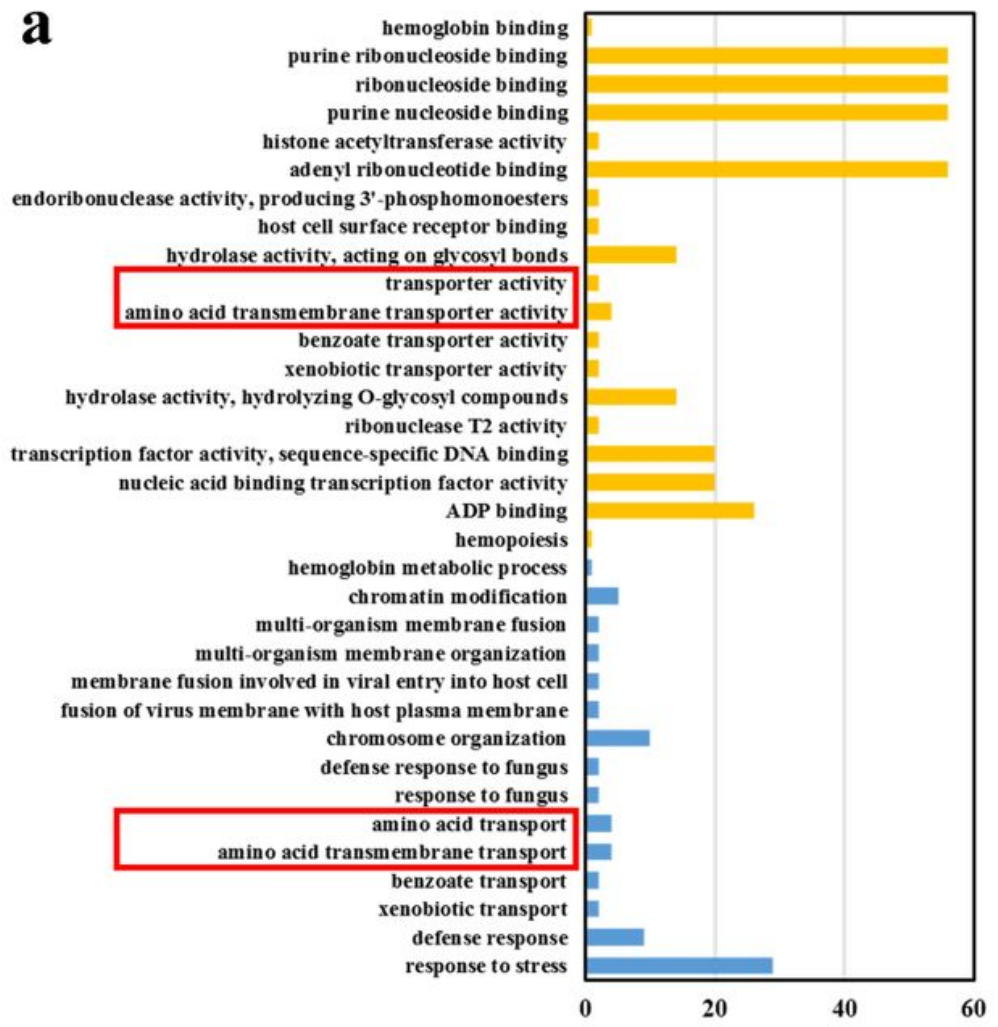

Biological process Molecular function b
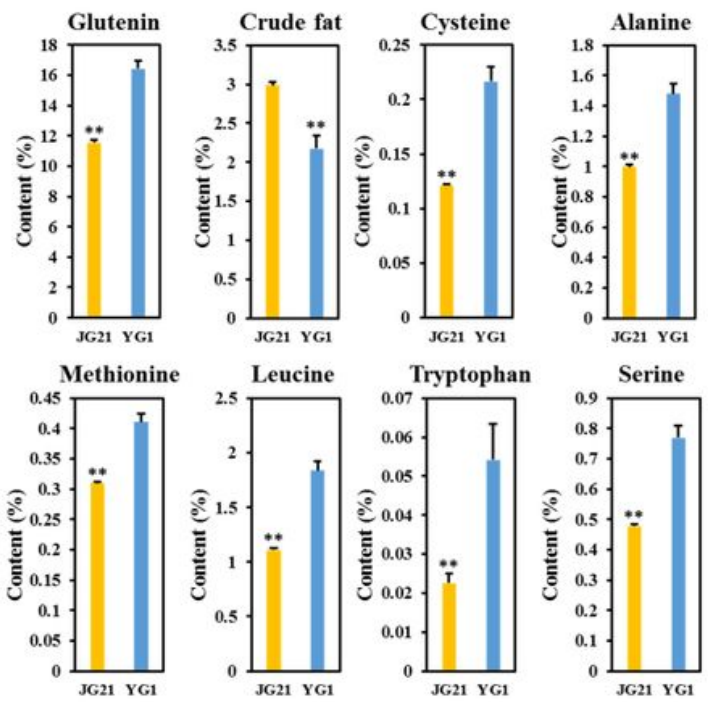

C

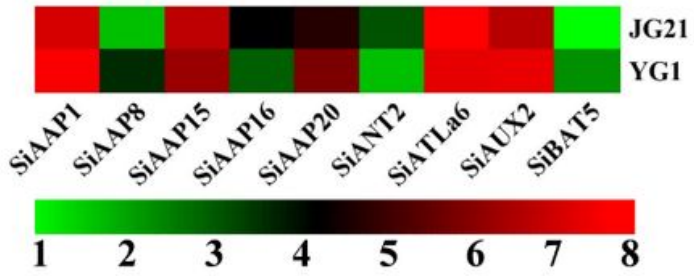


The comparative analysis on the transcriptome in grain development of two millet genotypes. (a) The GO enrichment of differently expressed genes (DEGs), the four terms related to amino acid transport were indicated with red boxes. (b) The differences in grain quality traits between "JG21" and "YG1". ** represents the differences significant at $P<0.01$. (c) The expression of 9 SiAATs in the grains of "JG21" and "YG1".

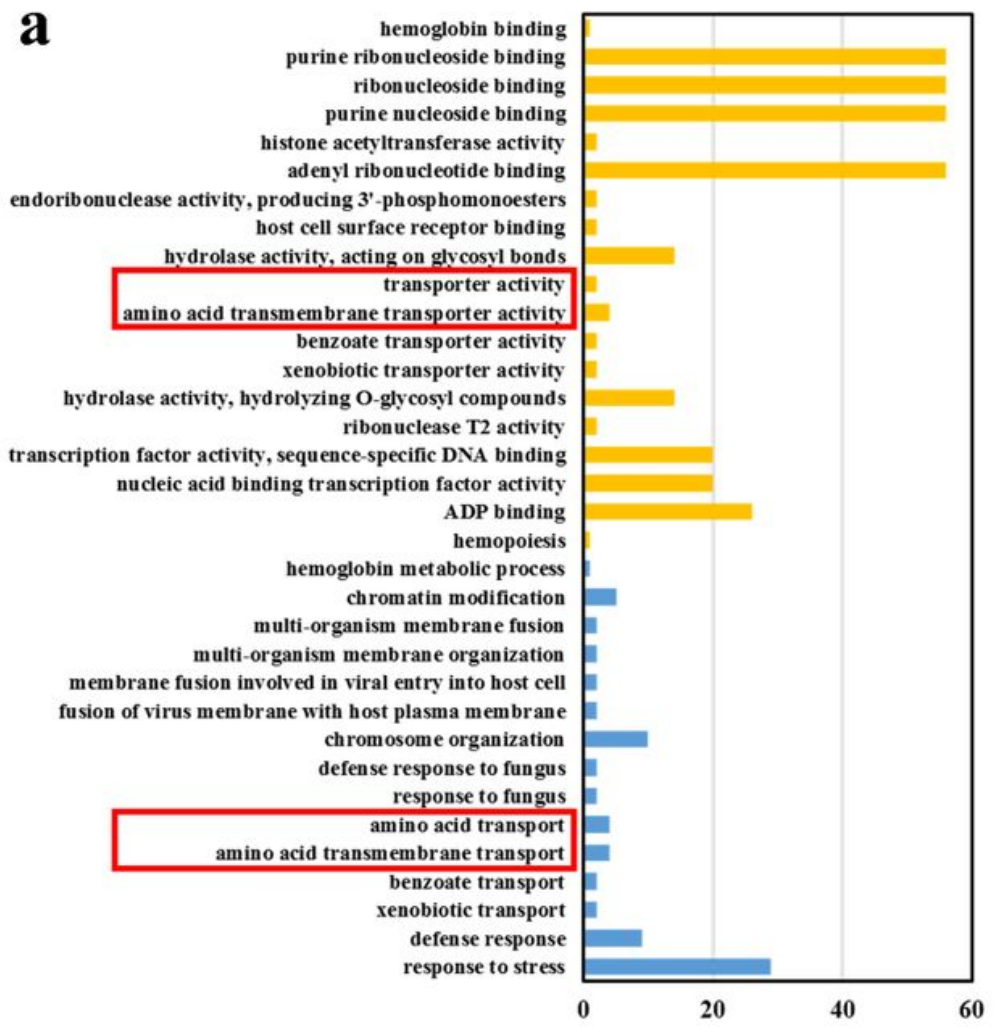

Biological process Molecular function b
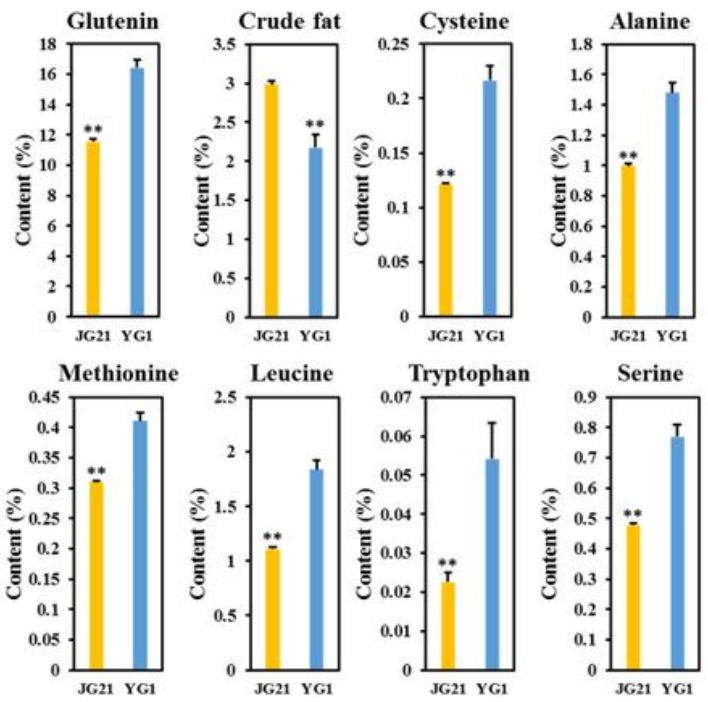

C

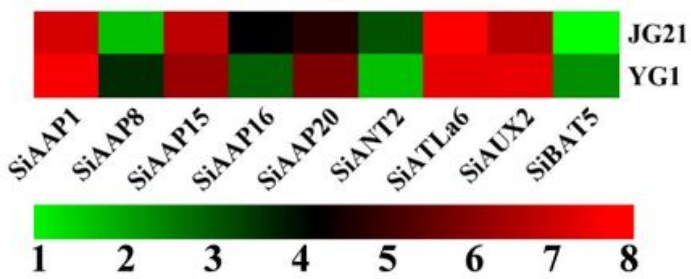

Figure 11

The comparative analysis on the transcriptome in grain development of two millet genotypes. (a) The GO enrichment of differently expressed genes (DEGs), the four terms related to amino acid transport were indicated with red boxes. (b) The differences in grain quality traits between "JG21" and "YG1". ** represents the differences significant at $P<0.01$. (c) The expression of 9 SiAATs in the grains of "JG21" and "YG1". 
$\mathbf{a}$

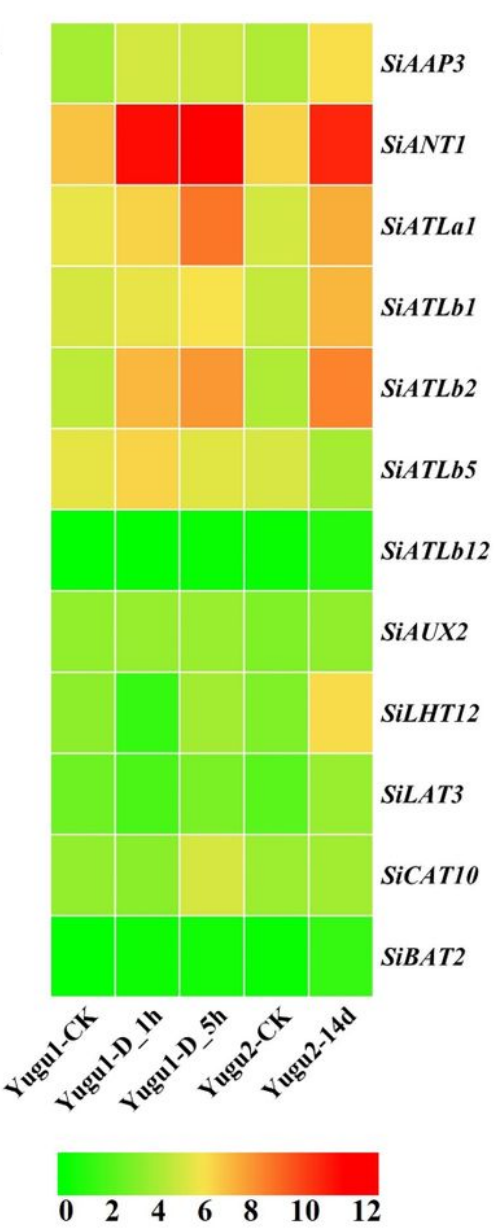

b
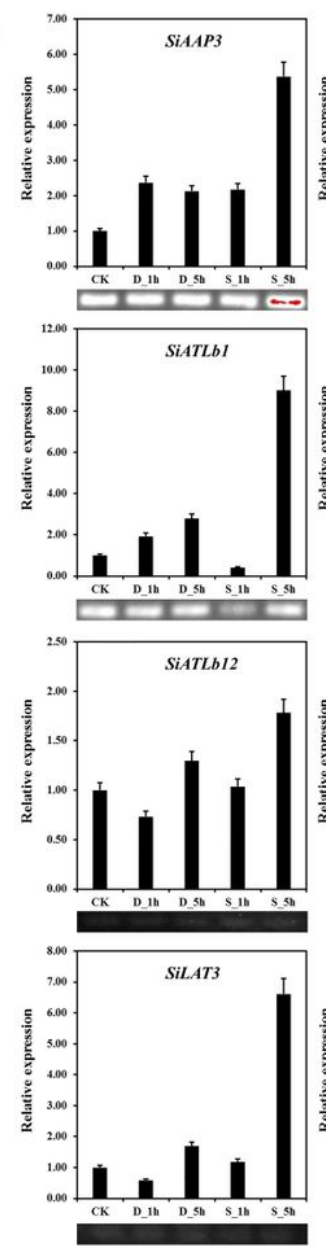
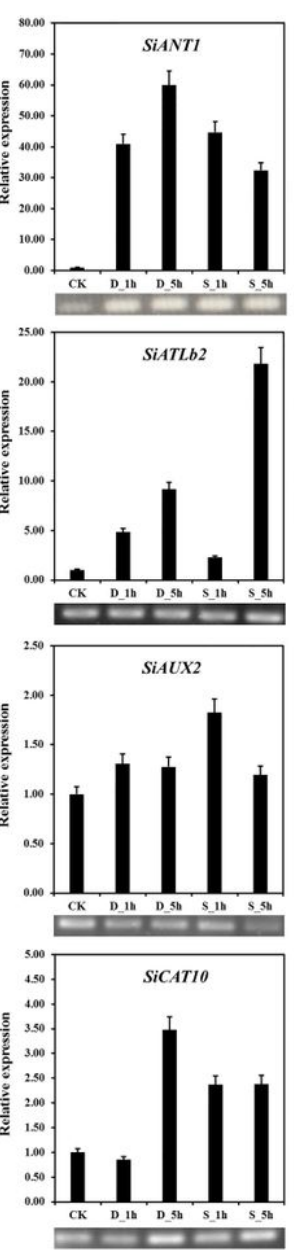
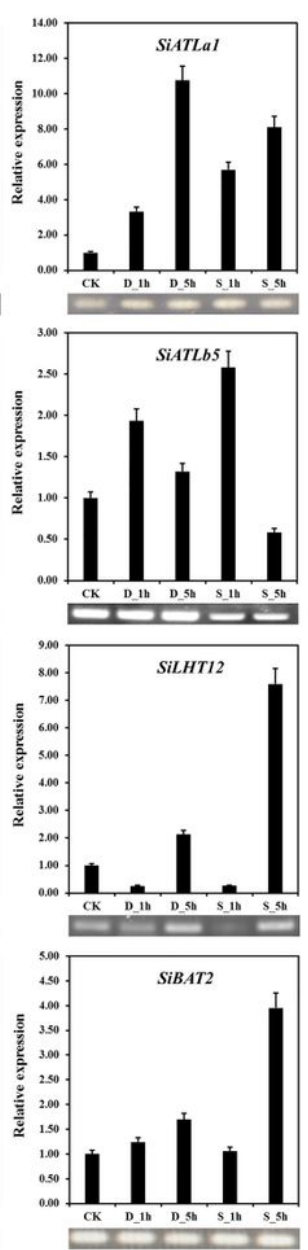

\section{Figure 12}

The expression patterns of 12 selected SiAAT genes under drought and salt stresses. (a) The RNA-seq results of 12 SiAATs under drought and salt stresses, which are reanalyzed with the public transcriptome data SRA062640 and PRJNA545871; (b) The qRT-PCR results of these 12 SiAATs. The seedlings 15 days after sowing were exposed to $20 \%$ polyethylene glycol (PEG 6000) solution and $\mathrm{NaCl}$ solution (200 mM) for $1 \mathrm{~h}$ and $5 \mathrm{~h}$, respectively. Bars represent the mean values of three replicates \pm standard deviation (SD). The SiAct-7 gene is used as an internal reference. The electropherograms of all PCR amplified products are also displayed in the corresponding position. 
a

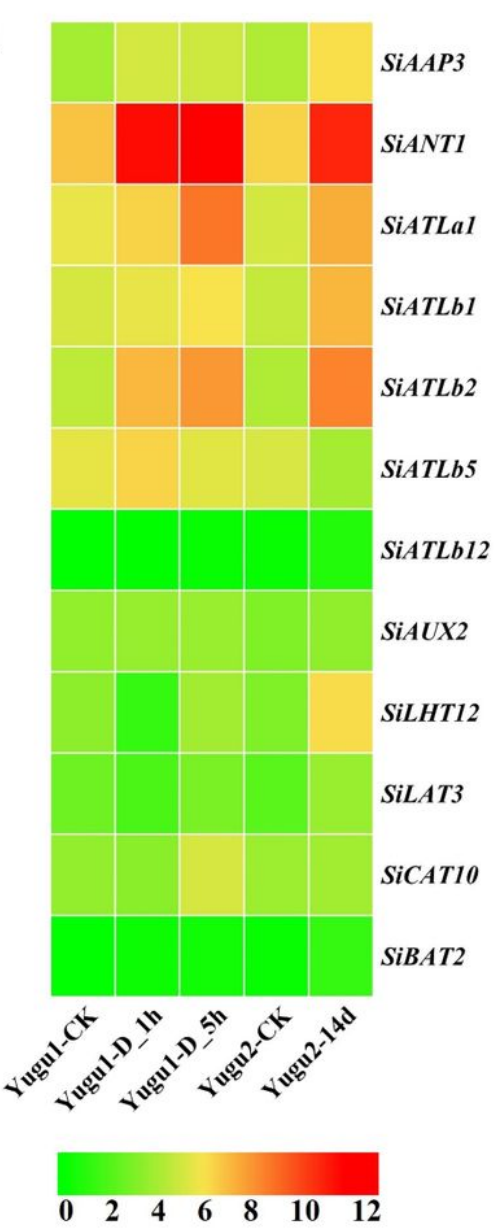

b
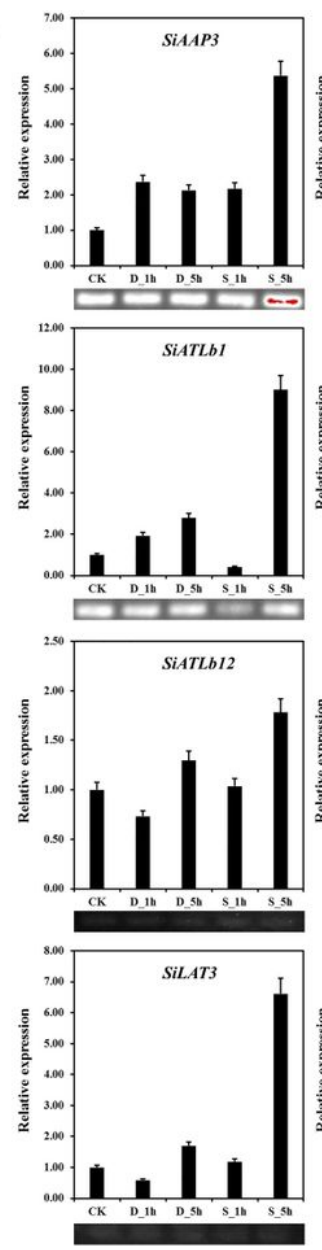
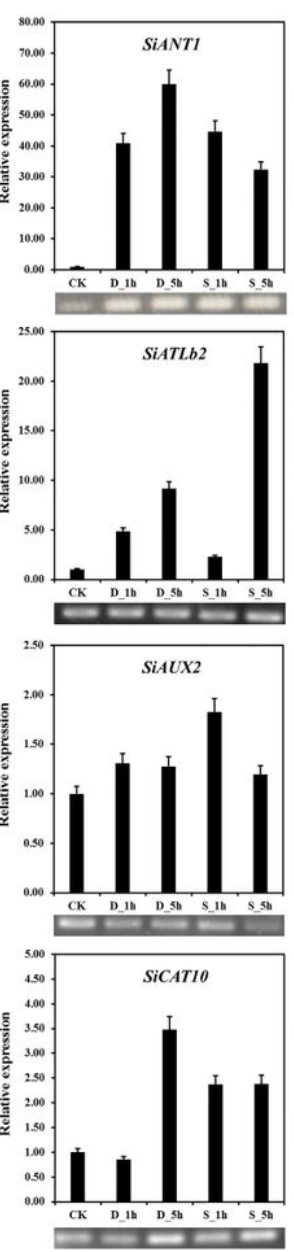
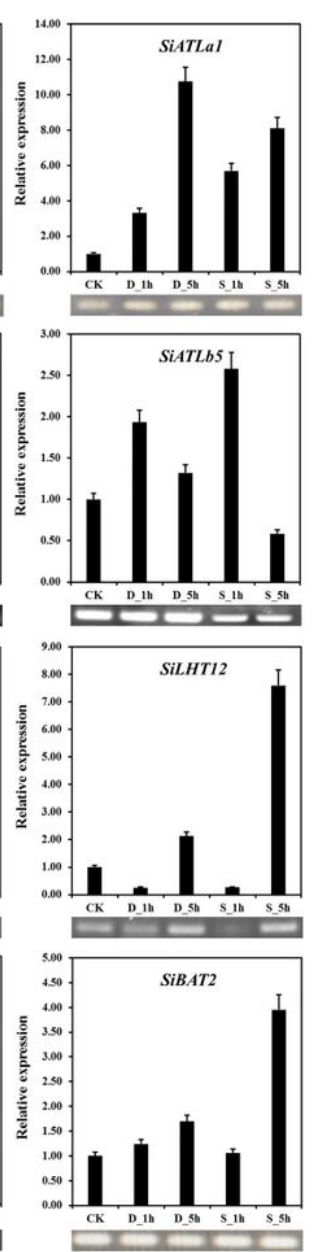

\section{Figure 12}

The expression patterns of 12 selected SiAAT genes under drought and salt stresses. (a) The RNA-seq results of 12 SiAATs under drought and salt stresses, which are reanalyzed with the public transcriptome data SRA062640 and PRJNA545871; (b) The qRT-PCR results of these 12 SiAATs. The seedlings 15 days after sowing were exposed to $20 \%$ polyethylene glycol (PEG 6000) solution and $\mathrm{NaCl}$ solution (200 mM) for $1 \mathrm{~h}$ and $5 \mathrm{~h}$, respectively. Bars represent the mean values of three replicates \pm standard deviation (SD). The SiAct-7 gene is used as an internal reference. The electropherograms of all PCR amplified products are also displayed in the corresponding position. 


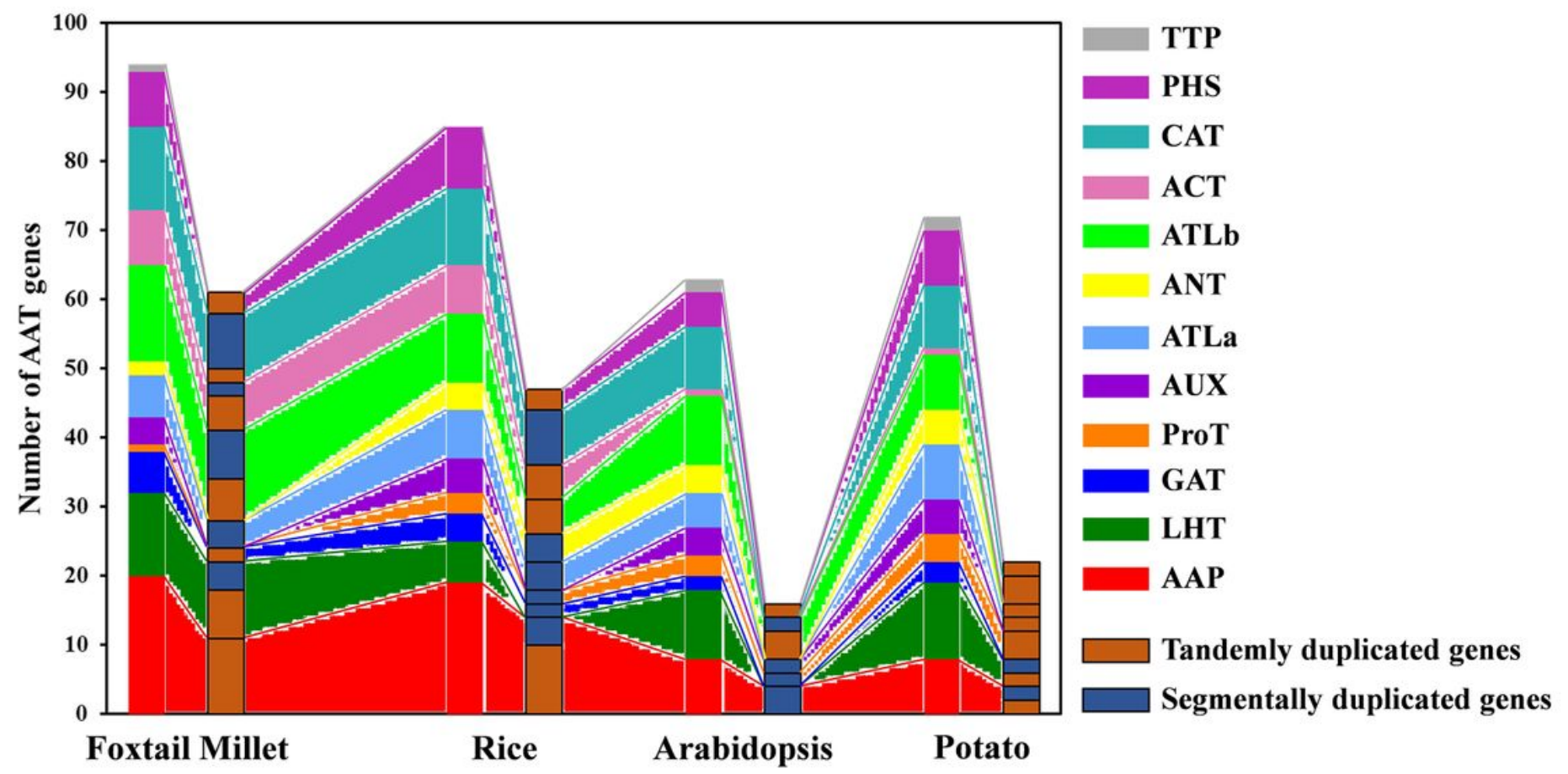

Figure 13

Distribution of the numbers of duplicated genes in different AAT subfamilies in foxtail millet, rice, Arabidopsis and potato. The number of all AAT genes and the number of AAT genes involved in the duplication event were in the column on the left and right of each species, respectively. The paralogous AAT genes generated by different duplication events and the AAT genes of different subfamily were marked with different colors. The same color connects the paralogous genes corresponding to the same subfamily.

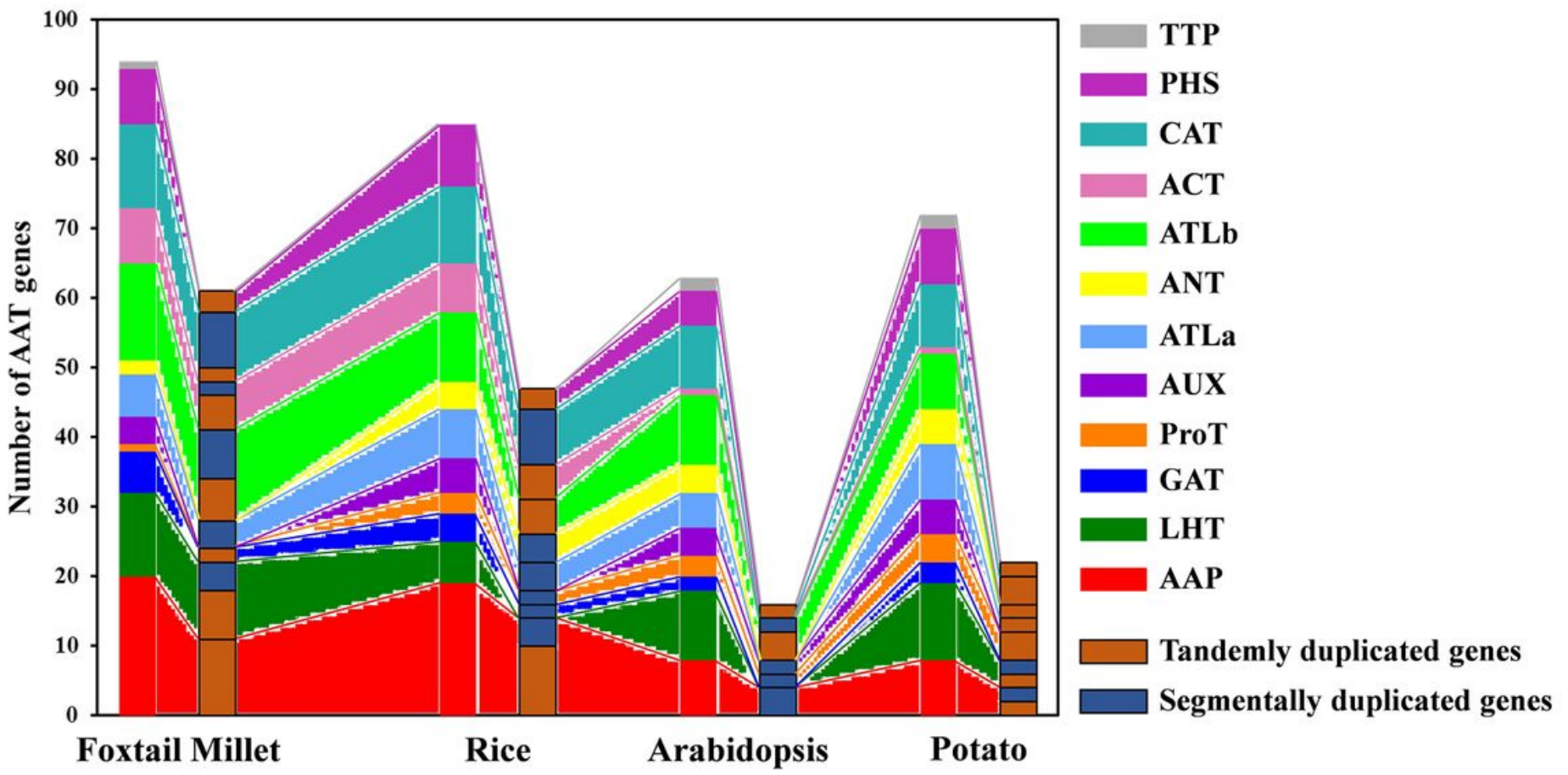

Figure 13

Distribution of the numbers of duplicated genes in different AAT subfamilies in foxtail millet, rice, Arabidopsis and potato. The number of all AAT genes and the number of AAT genes involved in the duplication event were in the column on the left and right of each species, respectively. The paralogous AAT genes generated by different duplication events and the AAT genes of different subfamily were marked with different colors. The same color connects the paralogous genes corresponding to the same subfamily. 


\section{Supplementary Files}

This is a list of supplementary files associated with this preprint. Click to download.

- FigureS1.jpg

- FigureS1.jpg

- Figures2.jpg

- Figures2.jpg

- FigureS3.jpg

- Figures3.jpg

- FigureS4.jpg

- FigureS4.jpg

- Tables1.pdf

- Tables1.pdf

- Tables2.pdf

- Tables2.pdf

- Tables3.pdf

- Tables3.pdf 